\title{
Predicting ignition quality of oxygenated fuels using artificial neural networks
}

\begin{abstract}
:
Artificial intelligence based computing systems like artificial neural networks (ANN) have recently found increasing applications in predicting complex chemical phenomena like combustion properties. The present work deals with the development of an ANN model which can predict the derived cetane number (DCN) of oxygenated fuels containing alcohol and ether functionalities. Experimental DCN's of 499 fuels comprising of 116 pure compounds, 222 pure compound blends, and 159 real fuel blends were used as the dataset for model development. DCN measurements of sixty new fuels were carried out in the present work and the data for the rest was collected from the literature. Fuel chemical composition expressed in the form of eight functional groups namely paraffinic $\mathrm{CH}_{3}$ groups, paraffinic $\mathrm{CH}_{2}$ groups, paraffinic $\mathrm{CH}$ groups, olefinic $-\mathrm{CH}=\mathrm{CH}_{2}$ groups, naphthenic $\mathrm{CH}-\mathrm{CH}_{2}$ groups, aromatic $\mathrm{C}-\mathrm{CH}$ groups, alcoholic $\mathrm{OH}$ groups and ether $\mathrm{O}$ groups, along with two structural parameters namely, molecular weight and branching index (BI), were used as the ten input features of the model. The qualitative and quantitative determination of functional groups present in real fuels was performed using ${ }^{1} \mathrm{H}$ nuclear magnetic resonance (NMR) spectroscopy. A robust ANN methodology was followed to prevent over fitting using a multilevel grid search and genetic algorithm. The final developed model with two hidden layers was tested with $15 \%$ of randomly generated unseen points from the dataset and a regression coefficient $\left(R^{2}\right)$ of 0.992 was observed between the experimental and predicted DCN values. An average absolute error of 0.91 obtained from the test set indicates that the developed ANN model is successful in predicting the DCN of oxygenated fuels and captures the dependence of the fuel's ignition quality (i.e.
\end{abstract} DCN) on its constituent functional groups.

Keywords: Derived Cetane Number (DCN); functional group; ${ }^{1} \mathrm{H}$ NMR spectroscopy; ANN;

\section{INTRODUCTION}

Combustion devices are the primary power generators used in the transportation sector; and will continue to be in the foreseeable future. Due to the ease of transportation, storage, handling, and, most importantly, high energy densities, liquid fuels are preferred sources of energy in all sectors of transportation. In recent years, there has been a great push in electrification of transport due to pollutant emissions and climate change concerns. Many studies [1,2] indicate that forms of electrification will be mainly concentrated in light duty vehicles (LDV). However, heavy duty vehicles (HDV), marine engines and jet engines are more difficult to electrify [2]. These trends are likely to shift the demand in liquid fuels in transportation sector to the diesel and jet fuel applications [3]. 
Compression ignition $(\mathrm{Cl})$ engines have no external ignition source and depend on the rise in pressure and temperature due to compression of the upward moving piston to ignite the in-cylinder fuel-air mixture. Conventional $\mathrm{Cl}$ engines operate with diesel fuels with high chemical reactivity, which is characterized by their short ignition delay times (IDTs). Similar to the octane scales for gasoline fuels [4], the cetane scale [5] was developed to quantify diesel fuel reactivity using the cetane number (CN).

The first technique used to determine the $\mathrm{CN}$ of a fuel relied on engine experiments in a Cooperative Fuel Research (CFR) F5 engine based on the ASTM D613 standard [5], which had inherent difficulties and was a time consuming process. For easier and more controlled $\mathrm{CN}$ determination, a constant volume combustion chamber (CVCC) known as the ignition quality tester (IQT) was designed and standardized [6] in the late 1990s. The IQT measures the IDT of an injected liquid fuel spray which then enables the calculation of the derived cetane number (DCN). The calculated DCN is functionally equivalent to the $\mathrm{CN}$ measurement from a CFR engine and will be collectively termed as $D / C N$, hereafter. However, it is to be noted that the autoignition conditions in the IQT and CFR are not the same. The CN measurements in the CFR engine based on the ASTM D613 standard relies on a variable compression ratio approach. This variation in compression ratio in a CFR engine results in different pressure-temperature history for different fuels compared to the IQT that has constant initial pressure and temperature condition. Since the pressure-temperature condition in the IQT and the correlation between DCN-IDT remains the same for every fuel it is reasonable to assume that the DCN represents accurate autoignition behavior of fuels with different chemistries.

Initially, the $\mathrm{D} / \mathrm{CN}$ measurement was limited to diesel fuels; however, recently several groups across the world have extended it to a broad spectrum of fuels [7-9]. As the ideal fuel for low temperature combustion (LTC) engines are likely to have auto-ignition quality in between diesel and gasoline; the octane and cetane scales have been correlated [10]. Recently a study [11] has also indicated the importance of DCN in understanding lean blow out (LBO) limits in gas turbine/jet engine applications. The LBO limit is critical parameter in combustion design for gas turbine applications, as the need for higher fuel efficiencies are pushing operation to lean limits where blow out in combustors can occur.

Diesel fuels are typically a mixture of petroleum-derived hydrocarbons. Oxygenated fuels have garnered interest as suitable blending agents with diesel fuels [12-15] for $\mathrm{Cl}$ engine applications as they are derived from renewable biomass sources [16-18] and due to their low sooting tendencies [19-23]. These oxygenated fuels include alcohols [24-29], ethers [12-14,30,31], esters [30,32] etc. Prediction of 
DCN of pure fuels (both oxygenated and non-oxygenated) have been a focus of many recent studies [16,33-39]. Although engine operation with pure oxygenated fuels is possible, the use of pure oxygenated fuels in $\mathrm{Cl}$ engine applications may be limited due to fuel compatibility issues such as, poor lubricating properties, corrosiveness, and low energy density [57]. Thus it is imperative to understand and be able to predict the behavior of oxygenated fuels when blended with conventional fuels such as gasolines and diesel in order to understand the synergistic/antagonistic effects on DCN for these fuel/oxygenate blends [40].

In general, DCN measurements of pure compounds are less time consuming compared to conventional engine measurements; however, the DCN measurements of fuel/oxygenate blends can be time consuming when compositional sweeps with multiple binary, ternary, and quaternary mixtures must be done. In such cases, it would be advantageous to predict DCN and blending behavior using models, which could be extended to multiple fuel/oxygenate combinations, which otherwise would be difficult with IQT experiments. With the importance of DCN in the IC engine community and the growing importance of DCN in the gas turbine/jet engine community, DCN prediction with mathematical models and/or machine learning models is needed and advantageous for fuel screening purposes. The development of such a machine learning model is the motivation of the present work.

Several methods and correlations have been developed to predict ignition quality (i.e. D/CN) of n-paraffins [41], pure hydrocarbons [35,37,42-44], blends of pure hydrocarbons [37], oxygenated hydrocarbons [34,39,45-47], diesel fuels [36,37,48-58], synthetic diesels [59], biofuel candidates [33], furanic biofuel additives [16] and biodiesel [38,60-62]. The D/CN prediction models reported in the literature have used both physical and chemical properties of the fuel as model inputs. Physical properties like density [36], viscosity [63], distillation characteristics [36] and aniline point [57] have been used. Molecular structure based techniques like quantitative structure property relationships (QSPR) [16,34,35,42,44], group contribution [39,43], hydrocarbon lumps [54], functional groups $[37,58,59,64]$, carbon groups [53] and principal component analysis [45], have also been employed for predicting D/CN. Analytical techniques such as nuclear magnetic resonance (NMR) spectroscopy $[37,45,51,53,58,65]$, gas chromatography coupled with mass spectroscopy (GC-MS) [56], near infra-red spectroscopy (NIR) [50] and high performance liquid chromatography (HPLC) [51] have also found applications in developing chemical composition based techniques for $\mathrm{D} / \mathrm{CN}$ prediction.

Fuels can be also be characterized by their relatively small number of molecular moieties, or functional groups, which dictate the combustion characteristics of the fuel like octane number [66], CN 
[37], flash point [34], sooting tendencies [20], auto-ignition [67], ignition delay [68] etc. Recent works by Abdul Jameel et al. $[69,70]$ and Mueller $[71,72]$, have shown that formulating simple surrogates by mimicking the functional groups in the fuel gives the ability to reproduce various physical, thermochemical and combustion properties of the fuel. This approach called as the minimalist functional group (MFG) approach has been comprehensively tested on a number of gasoline, diesel and jet fuels $[69,70,73]$. A number of other studies $[21,22,64,74-78]$ reported in the literature have also hinted at the ability of functional groups to reproduce fuel behavior. Therefore, expressing a fuel's chemical composition by means of functional groups enables us to capture its chemical nature and predict its DCN. ${ }^{1} \mathrm{H}$ NMR spectroscopy is an advanced analytical technique that can qualitatively and quantitatively estimate the fuels functional groups as shown in a number of works [79-84]. Oxygenated fuels containing alcohol $\mathrm{OH}$ and ether $\mathrm{O}$ functionalities can be divided into eight functional groups namely paraffinic $\mathrm{CH}_{3}$ groups, paraffinic $\mathrm{CH}_{2}$ groups, paraffinic $\mathrm{CH}$ groups, olefinic $-\mathrm{CH}=\mathrm{CH}_{2}$ groups, naphthenic $\mathrm{CH}-\mathrm{CH}_{2}$ groups, aromatic $\mathrm{C}-\mathrm{CH}$ groups, alcoholic $\mathrm{OH}$ groups and ether $\mathrm{O}$ groups and serve as inputs to develop a DCN model. Physical phenomenon such as fuel vaporization and spray formation are encountered during an IQT measurement and affect the subsequent gas phase combustion process. These physical phenomenon can be correlated to the molecular weight of the fuel and alkyl substitution as shown in detail in previous works $[37,66]$. Therefore, two additional structural properties namely molecular weight and branching index (BI) serve as additional input features along with the abovementioned eight functional groups. More information on $\mathrm{BI}$ is presented in section 3.1. Together these ten parameters possess enough fuel information to be able to predict its autoignition propensity (i.e. DCN) $[37,64]$.

Mathematical techniques such as multiple linear regression (MLR) $[49,58,60,85]$ and partial least squares (PLS) [50] have been applied to analyze the compositional data and then deployed as tools to develop mathematical models to predict $\mathrm{CN}$. These techniques have been used due to the simplicity and ease associated with model development and application. The predictive capability of these linear techniques is limited especially when applied to diverse samples from the dataset and there is the inherent inability to capture complex non-linear relationship, which is so often noticed in chemical phenomenon. Artificial neural networks (ANN) have the capacity to extract non-linear relationships between a set of inputs and output(s) and hence are ideally suited to grasp the synergistic/antagonistic relationships between functional groups so often observed [86-92]. 
The present work is an extension of our previous MLR based DCN model [37] developed for hydrocarbon fuels applicable to pure compounds, blends of pure compounds, and real fuels. In this work, an ANN model was used to correlate the non-linear relationship between the ten input features (eight functional groups, molecular weight and $\mathrm{BI}$ ) and DCN, and the developed model was then used to predict the DCN of oxygenated fuels containing alcohol $\mathrm{OH}$ and ether $\mathrm{O}$ functionalities. A dataset of 499 fuels consisting of 116 pure compounds, 222 pure compound blends and 159 real fuel blends was used. The novelty of this work is that it uses extensive data that possesses the synergistic/antagonistic DCN behaviors of a number of fuels mixtures and the robust ANN methodology used for model development is able to capture this behavior due to the use of the input parameters. The weight $\%$ of the various functional groups in the pure compounds was calculated from their molecular structure and those in the real fuels was determined using ${ }^{1} \mathrm{H}$ NMR spectroscopy. DCN of these fuels was experimentally obtained using an IQT for many of the fuels and blends, and gathered from the literature for the remaining.

\section{EXPERIMENTAL METHODS}

\subsection{Nuclear Magnetic Resonance Spectroscopy}

${ }^{1}$ H NMR measurements of two naphtha's (light and heavy; LN J80 and HN J80) and one Satorp gasoline (SG) were performed in the present work and the ${ }^{1} \mathrm{H}$ NMR spectral data of the other real fuels reported here were taken from previous studies $[37,69]$. Each sample was prepared by dissolving $100 \mu \mathrm{l}$ of the fuel in $600 \mu \mathrm{l}$ of deuterated chloroform $\left(\mathrm{CDCl}_{3}\right)$ and then $600 \mu \mathrm{l}$ of the mixture was transferred to $5 \mathrm{~mm}$ NMR tubes. A Bruker $700 \mathrm{MHz}$ AVANACIII NMR spectrometer equipped with Bruker CPTCI multinuclear CryoProbe (BrukerBioSpin, Rheinstetten, Germany) was used for all ${ }^{1} \mathrm{H}$ NMR measurements. All spectra were collected under the same conditions using same instrumental parameters as described in previous works [37,69]. Bruker Topspin 4.0.4 software was used for data collection and MestReNova was used for spectra post processing and data analyses.

\subsection{Ignition quality tester}

The DCN of pure compounds, pure hydrocarbon blends, pure hydrocarbon-oxygenate blends and real fuel-oxygenate blends are presented in Tables 1, 2, 3 and 4 respectively, along with the standard deviation (SD) and standard error (SE) when available. DCN measurements of sixty fuel blends comprising of gasoline primary reference fuels (PRF) (mixtures of n-heptane and 2,2,4trimethylpentane), toluene primary reference fuels (TPRF), methanol, ethanol, n-propanol, n-butanol, 2methylbutan-1-ol, diethyl ether (DEE), dipropyl ether (DPE), diisopropyl ether (DIPE), SG, LN J80 and HN 
J80 were experimentally measured in the IQT following ASTM D6890 standard [6] (see Tables 3 and 4) and the DCN of the rest of the fuels were obtained from the literature.

The IQT is pressurized with zero air (purified air with less than $0.1 \mathrm{ppm}$ of total hydrocarbons) and then the temperature is raised to a standard temperature prescribed by the ASTM D6890 standard. The CVCC pressure is set to 21.3 bar and the standard temperature is in the range of $830-840 \mathrm{~K}$. IDT is calculated as the time period between the start of fuel injection (Solnj) when the pintle injector opens and the sharp rise in pressure as a result of ignition. The ignition delay time used in the present work is obtained with the gradient method that is described in detail here [93]. The gradient method gives different IDTs compared to ASTM D6890 method only for fuels that exhibit strong low temperature heat release (LTHR). For diesel-like fuels due to the shorter time scales LTHR is not conspicuous and both the methods (gradient and ASTM D6890) provided similar IDTs. For DCN values obtained from literature, no indication of LTHR has been explicitly stated; hence, if LTHR exist the IDT would be slightly shorter than that obtained using the gradient method. However, due to the power law nature of the correlation between DCN and IDT as given by Equation 1, if LTHR exists the difference in DCN with both methods would be minimal at long IDTs (since slopes approach zero).

As prescribed by the standard, 15 pre-injections are performed to stabilize and provide a uniform and steady combustion environment for the subsequent 32 main injections that are logged. The IDT obtained for each fuel blend is the average of 32 main injections. The average IDT is then used to obtain the DCN using the correlations given by Equations 1 and 2, which are obtained from the ASTM D6890 standard. The appropriate correlation to be used is based on the range of the IDT (in ms); for all IDTs in the range of 3.1-6.5 ms, Equation 1 is used; for all values outside this range, Equation 2 is used.

$D C N=4.46+\frac{186.6}{\tau[m s]}$

$D C N=83.99(\tau[m s]-1.512)^{-0.658}+3.547$

For low DCN (<34) the DCN-IDT correlation given by Eq. (2) is less precise compared to the standard DCN range of 34--65 (Eq. (1)). However, compared to high DCN (> 65) the precision in the lower DCN range is better as the reproducibility and repeatability errors are proportional to DCN as given by Equations 3 and 4, which are obtained from the ASTM D6890 standard.

Repeatability $=0.01215 \times(\mathrm{DCN}+3.5)$

Reproducibility $=0.06201 \times($ DCN -13.7$)$ 
Alternatively, due to the power law nature of the correlation between DCN and IDT (Equation 2) the errors in DCN determination (for low DCN values) would be minimal at long IDTs (since slopes approach zero [39]). In our study, the DCNs of a major portion of the pure components and real fuel blends lie in the range of 10--35; a range we are confident in the DCNs compared to high DCNs that are subject to uncertainties due to large variation in DCN for a small change in IDT.

\section{Table 1}

$\mathrm{D} / \mathrm{CN}$ of pure compounds

\begin{tabular}{|c|c|c|c|c|}
\hline S. No. & Name & $(\mathrm{D} / \mathrm{CN})$ & $S D$ & $S E$ \\
\hline & PARAFFINS & & & \\
\hline 1 & n-butane & 20.6 & 0.24 & 0.04 \\
\hline 2 & $\mathrm{n}$-pentane & 39.4 & & \\
\hline 3 & n-hexane & 50 & & \\
\hline 4 & n-heptane & 53.8 & 0.61 & 0.11 \\
\hline 5 & n-octane & 58.2 & & \\
\hline 6 & n-nonane & 60.9 & & \\
\hline 7 & n-decane & 65.5 & & \\
\hline 8 & n-dodecane & 72.9 & & \\
\hline 9 & n-tetradecane & 85.1 & & \\
\hline \multirow[t]{2}{*}{10} & n-hexadecane & 100 & & \\
\hline & ISO-PARAFFINS & & & \\
\hline 11 & 2-methylbutane & 25.8 & 0.76 & 0.13 \\
\hline 12 & 2-methylpentane & 34.5 & & \\
\hline 13 & 3-methylpentane & 30.7 & 0.62 & 0.11 \\
\hline 14 & 2,2-dimethylbutane & 24.4 & & \\
\hline 15 & 2-methylhexane & 43.5 & & \\
\hline 16 & 3-methylhexane & 42.0 & 0.51 & 0.09 \\
\hline 17 & 3-ethylpentane & 34.1 & & \\
\hline 18 & 2,4-dimethylpentane & 28.2 & 0.44 & 0.08 \\
\hline 19 & 2,3-dimethylpentane & 21.7 & 0.46 & 0.08 \\
\hline 20 & 2,2,3-trimethylbutane & 12.9 & & \\
\hline 21 & 2-methylheptane & 52.6 & & \\
\hline 22 & 3-methylheptane & 45 & & \\
\hline 23 & 2,2,4-trimethylpentane & 17.4 & 0.38 & 0.07 \\
\hline 24 & 2,6-dimethyloctane & 51.7 & & \\
\hline 25 & 2,6,10-trimethyldodecane & 58 & & \\
\hline 26 & 2,6,10-trimethyltridecane & 60.8 & & \\
\hline 27 & $2,2,4,4,6,8,8$-heptamethylnonane & 15.1 & & \\
\hline 28 & 2-methylheptadecane & 91 & & \\
\hline \multirow[t]{2}{*}{29} & 2-methyloctadecane & 104.4 & & \\
\hline & OLEFINS & & & \\
\hline 30 & 2-methyl-1,3-butadiene & 13 & & \\
\hline 31 & 1-hexene & 28.9 & 0.59 & 0.10 \\
\hline 32 & 1-heptene & 32 & & \\
\hline 33 & 2,5-dimethyl-2,4-hexadiene & 19.9 & & \\
\hline 34 & 1-octene & 40 & & \\
\hline 35 & 3-octene & 36 & & \\
\hline 36 & 2,4,4-trimethyl-1-pentene & 8.8 & 0.21 & 0.04 \\
\hline 37 & 1-nonene & 51 & & \\
\hline 38 & 1-decene & 49.1 & & \\
\hline 39 & 1,9-decadiene & 41 & & \\
\hline 40 & 1-dodecene & 56.8 & & \\
\hline
\end{tabular}




\begin{tabular}{|c|c|c|c|c|}
\hline \multirow[t]{2}{*}{41} & 1-hexadecene & 75.9 & & \\
\hline & \multicolumn{4}{|l|}{ NAPHTHENES } \\
\hline 42 & Cyclopentane & 6.1 & & \\
\hline 43 & Methylcyclopentane & 17.2 & & \\
\hline 44 & Cyclohexane & 19.9 & 0.24 & 0.04 \\
\hline 45 & Cyclohexene & 18.1 & & \\
\hline 46 & Methylcyclohexane & 23.5 & & \\
\hline 47 & 1,2-dimethylcyclohexane & 24 & 0.39 & 0.07 \\
\hline 48 & 1,3-dimethylcyclohexane & 30.5 & 0.47 & 0.08 \\
\hline 49 & Ethylcyclohexane & 35.8 & & \\
\hline 50 & Cyclooctane & 22.3 & & \\
\hline 51 & 1,2,4-trimethylcyclohexane & 24.4 & & \\
\hline 52 & 1,3,5-trimethylcyclohexane & 30.5 & & \\
\hline 53 & n-buytlcyclohexane & 47.6 & & \\
\hline 54 & Decahydronaphthalene & 33 & & \\
\hline 55 & Perhydro-phenanthrene & 38.8 & & \\
\hline \multirow[t]{2}{*}{56} & 1,3,5-triisopropylcyclohexane & 25.3 & & \\
\hline & \multicolumn{4}{|l|}{ AROMATICS } \\
\hline 57 & Toluene & 6 & 0.21 & 0.04 \\
\hline 58 & Ethylbenzene & 6.29 & 0.12 & 0.02 \\
\hline 59 & 1,2-dimethylbenzene & 8.3 & 0.19 & 0.03 \\
\hline 60 & 1,3-dimethylbenzene & 7 & 0.23 & 0.04 \\
\hline 61 & 1,4-dimethyl benzene & 6.19 & 0.32 & 0.06 \\
\hline 62 & 1,2,3-trimethylbenzene & 10.14 & 0.10 & 0.02 \\
\hline 63 & 1,2,4-trimethylbenzene & 8.9 & 0.09 & 0.02 \\
\hline 64 & 1,3,5-trimethylbenzene & 8 & 0.13 & 0.02 \\
\hline 65 & n-butylbenzene & 12 & & \\
\hline 66 & Indan & 8.6 & 0.22 & 0.04 \\
\hline 67 & Tetralin & 9.4 & 0.31 & 0.05 \\
\hline 68 & 1-methylnaphthalene & 0 & & \\
\hline 69 & 1,3,5-triethylbenzene & 7.6 & 0.17 & 0.03 \\
\hline 70 & 1,3,5-triisopropylbenzene & 2.8 & & \\
\hline \multirow[t]{2}{*}{71} & 1,2-diphenylethane & 1 & & \\
\hline & \multicolumn{4}{|l|}{ ALCOHOLS } \\
\hline 72 & methanol & 6.1 & & \\
\hline 73 & ethanol & 8 & & \\
\hline 74 & n-propanol & 8.2 & & \\
\hline 75 & n-butanol & 11.7 & & \\
\hline 76 & 2-methylbutan-1-ol & 11.36 & & \\
\hline 77 & 2-methoxyethanol & 15.5 & & \\
\hline 78 & 2-butanol & 8.14 & & \\
\hline 79 & 2-methylpropan-2-ol & 8.11 & & \\
\hline 80 & 1-methoxy-2-propanol & 16.3 & & \\
\hline 81 & cyclopentanol & 9.8 & & \\
\hline 82 & 2-methyl-2-butanol & 12.1 & & \\
\hline 83 & 3-methyl-1-butanol & 12.8 & & \\
\hline 84 & 2-isopropoxyethanol & 27.8 & & \\
\hline 85 & cyclohexanol & 16.3 & & \\
\hline 86 & 3-methoxy-3-methyl-1-butanol & 10 & & \\
\hline 87 & 1-heptanol & 29 & & \\
\hline 88 & 2-heptanol & 25 & & \\
\hline 89 & 4-heptanol & 21 & & \\
\hline 90 & guaiacol & 19.3 & & \\
\hline 91 & 2-phenylethanol & 8 & & \\
\hline 92 & 4-methyl guaiacol & 19.8 & & \\
\hline 93 & 2,6-dimethoxyphenol & 26 & & \\
\hline 94 & 1-octanol & 33.9 & & \\
\hline 95 & 3-octanol & 25.1 & & \\
\hline
\end{tabular}




\begin{tabular}{llc}
96 & 2-ethyl-1-hexanol & 23.5 \\
97 & 4-propyl phenol & 8.6 \\
98 & 1-nonanol & 46.2 \\
99 & 2-nonanol & 39.6 \\
100 & 4-nonanol & 28 \\
& ETHERS & \\
101 & 1,2-dimethoxyethane & 81 \\
102 & tetrahydrofuran & 21.9 \\
103 & 2-methyl tetrahydrofuran & 21.3 \\
104 & tetrahydropyran & 38.2 \\
105 & 2,2-dimethoxypropane & 31.3 \\
106 & Diethylether & 139 \\
107 & Dibutylether & 115.2 \\
108 & Dimethoxymethane & 29.9 \\
109 & cyclopentyl methyl ether & 47.3 \\
110 & 2-ethyl tetrahydrofuran & 28.1 \\
111 & diisopropyl ether & 23.6 \\
112 & anisole & 6.4 \\
113 & hexylmethyl ether & 99.8 \\
114 & 1-butoxy-2-propanol & 36.1 \\
115 & diisobutylether & 59.7 \\
116 & 1,2-dimethoxybenzene & 17 \\
\hline & Entries 1-71 : Ref: [37]
\end{tabular}

Entries 72-116 : Ref: [8] 
Table 2

DCN of pure hydrocarbon blends.

\begin{tabular}{|c|c|c|c|c|c|c|c|c|c|c|c|c|}
\hline \multirow[t]{2}{*}{ S.no } & \multicolumn{9}{|c|}{ Components (vol \%) } & \multirow[t]{2}{*}{ DCN } & \multirow[t]{2}{*}{ SD } & \multirow[t]{2}{*}{ SE } \\
\hline & n-heptane & iso-octane & n-hexadecane & 1-hexene & cyclopentane & cyclohexane & toluene & $\begin{array}{l}1,2,4- \\
\text { trimethylbenzene }\end{array}$ & tetralin & & & \\
\hline 1 & 50 & 50 & & & & & & & & 40.1 & 0.59 & 0.10 \\
\hline 2 & 40 & 60 & & & & & & & & 36.1 & 0.53 & 0.09 \\
\hline 3 & 30 & 70 & & & & & & & & 31.6 & 0.41 & 0.07 \\
\hline 4 & 20 & 80 & & & & & & & & 27.7 & 0.55 & 0.10 \\
\hline 5 & 10 & 90 & & & & & & & & 23.3 & 0.70 & 0.12 \\
\hline 6 & & 90 & & 10 & & & & & & 21.02 & 0.38 & 0.07 \\
\hline 7 & & 80 & & 20 & & & & & & 21.17 & 0.69 & 0.12 \\
\hline 8 & & 70 & & 30 & & & & & & 22.90 & 0.68 & 0.12 \\
\hline 9 & & 60 & & 40 & & & & & & 24.02 & 0.83 & 0.15 \\
\hline 10 & & 50 & & 50 & & & & & & 24.51 & 0.66 & 0.12 \\
\hline 11 & & 40 & & 60 & & & & & & 25.34 & 0.39 & 0.07 \\
\hline 12 & & 30 & & 70 & & & & & & 26.50 & 0.47 & 0.08 \\
\hline 13 & & 20 & & 80 & & & & & & 27.20 & 0.44 & 0.08 \\
\hline 14 & & 10 & & 90 & & & & & & 28.20 & 0.76 & 0.13 \\
\hline 15 & & 90 & & & & 10 & & & & 18.12 & 0.67 & 0.12 \\
\hline 16 & & 80 & & & & 20 & & & & 18.20 & 0.39 & 0.07 \\
\hline 17 & & 70 & & & & 30 & & & & 18.28 & 0.19 & 0.03 \\
\hline 18 & & 60 & & & & 40 & & & & 18.34 & 0.42 & 0.07 \\
\hline 19 & & 50 & & & & 50 & & & & 18.43 & 0.55 & 0.10 \\
\hline 20 & & 40 & & & & 60 & & & & 18.69 & 0.29 & 0.05 \\
\hline 21 & & 30 & & & & 70 & & & & 19.04 & 0.46 & 0.08 \\
\hline 21 & & 20 & & & & 80 & & & & 19.45 & 0.25 & 0.04 \\
\hline 23 & & 10 & & & & 90 & & & & 19.68 & 0.31 & 0.05 \\
\hline 24 & 60 & & & & & & 40 & & & 36.7 & 0.56 & 0.10 \\
\hline 25 & 55 & & & & & & 45 & & & 34.1 & 0.42 & 0.07 \\
\hline 26 & 50 & & & & & & 50 & & & 31.0 & 0.35 & 0.06 \\
\hline 27 & 45 & & & & & & 55 & & & 28.3 & 0.42 & 0.07 \\
\hline 28 & 40 & & & & & & 60 & & & 25.8 & 0.47 & 0.08 \\
\hline 29 & 35 & & & & & & 65 & & & 22.9 & 0.30 & 0.05 \\
\hline 30 & 30 & & & & & & 70 & & & 19.8 & 0.37 & 0.07 \\
\hline 31 & & 90 & & & & & & & 10 & 15.51 & 0.29 & 0.05 \\
\hline 32 & & 80 & & & & & & & 20 & 13.17 & 0.26 & 0.05 \\
\hline 33 & & 70 & & & & & & & 30 & 11.74 & 0.25 & 0.04 \\
\hline 34 & & 60 & & & & & & & 40 & 10.63 & 0.53 & 0.09 \\
\hline 35 & & 50 & & & & & & & 50 & 9.66 & 0.18 & 0.03 \\
\hline 36 & & 40 & & & & & & & 60 & 9.22 & 0.25 & 0.04 \\
\hline 37 & & 30 & & & & & & & 70 & 9.20 & 0.24 & 0.04 \\
\hline
\end{tabular}




$\begin{array}{lll}38 & & 20 \\ 39 & & 10 \\ 40 & 36 & 54 \\ 41 & 32 & 48 \\ 42 & 28 & 42 \\ 43 & 24 & 36 \\ 44 & 27 & 63 \\ 45 & 24 & 56 \\ 46 & 21 & 49 \\ 47 & 18 & 42 \\ 48 & 18 & 72 \\ 49 & 16 & 64 \\ 50 & 14 & 56 \\ 51 & 12 & 48 \\ 52 & 9 & 81 \\ 53 & 8 & 72 \\ 54 & 7 & 63 \\ 55 & 9 & 91 \\ 56 & 12.5 & 72.5 \\ 57 & 17.5 & 52.5 \\ 58 & 5.1 & 84.7 \\ 59 & 3 & 77 \\ 60 & 10 & 65 \\ 61 & 7 & 58 \\ 62 & 13.9 & 46.5 \\ 63 & 11 & 39 \\ 64 & 9 & 76 \\ 65 & 9 & 61 \\ 66 & 10 & 75 \\ 67 & 12.5 & 57.5 \\ 68 & 9 & 81 \\ 69 & 9 & 71 \\ 70 & 13 & 62 \\ 71 & 17 & 43 \\ 72 & 14 & 51 \\ 73 & 18 & 32 \\ 74 & 30 & 70 \\ 75 & 33 & 52 \\ 76 & 40 \\ 77 & 25 & 30 \\ & & 65\end{array}$

\begin{tabular}{|c|c|c|c|c|c|}
\hline & & 80 & 9.00 & 0.14 & 0.02 \\
\hline & & 90 & 8.92 & 0.27 & 0.05 \\
\hline & 10 & & 32.2 & 0.47 & 0.08 \\
\hline & 20 & & 29.1 & 0.26 & 0.05 \\
\hline & 30 & & 26.2 & 0.51 & 0.09 \\
\hline & 40 & & 22.7 & 0.35 & 0.06 \\
\hline & 10 & & 28.9 & 0.44 & 0.08 \\
\hline & 20 & & 26.3 & 0.39 & 0.07 \\
\hline & 30 & & 23.7 & 0.23 & 0.04 \\
\hline & 40 & & 19.9 & 0.22 & 0.04 \\
\hline & 10 & & 25.4 & 0.27 & 0.05 \\
\hline & 20 & & 22.8 & 0.25 & 0.04 \\
\hline & 30 & & 19.8 & 0.18 & 0.03 \\
\hline & 40 & & 16.5 & 0.14 & 0.02 \\
\hline & 10 & & 21.1 & 0.80 & 0.14 \\
\hline & 20 & & 18.3 & 0.43 & 0.08 \\
\hline & 30 & & 15.6 & 0.27 & 0.05 \\
\hline & & & 23.23 & 0.27 & 0.05 \\
\hline & 15 & & 21.8 & 0.34 & 0.06 \\
\hline & 30 & & 21.6 & 0.45 & 0.08 \\
\hline 10.2 & & & 21.34 & 0.60 & 0.11 \\
\hline 20 & & & 21.07 & 0.40 & 0.07 \\
\hline 10 & 15 & & 21.6 & 0.45 & 0.08 \\
\hline 20 & 15 & & 21.12 & 0.28 & 0.05 \\
\hline 7.7 & 31.9 & & 20.94 & 0.42 & 0.07 \\
\hline 20 & 30 & & 21.05 & 0.45 & 0.08 \\
\hline & & 15 & 20.96 & 0.36 & 0.06 \\
\hline & & 30 & 19.2 & 0.48 & 0.08 \\
\hline & 8 & 7 & 21.36 & 0.47 & 0.08 \\
\hline & 15 & 15 & 20.34 & 0.28 & 0.05 \\
\hline 10 & & & 19.45 & 0.32 & 0.06 \\
\hline 20 & & & 17 & 0.47 & 0.08 \\
\hline 10 & 15 & & 19.51 & 0.44 & 0.08 \\
\hline 10 & 30 & & 20.12 & 0.38 & 0.07 \\
\hline 20 & 15 & & 17.83 & 0.44 & 0.08 \\
\hline 20 & 30 & & 18.05 & 0.36 & 0.06 \\
\hline & & & 31.6 & 0.55 & 0.10 \\
\hline & 15 & & 33.24 & 0.43 & 0.08 \\
\hline & 30 & & 32.47 & 0.51 & 0.09 \\
\hline 10 & & & 30.97 & 0.39 & 0.07 \\
\hline
\end{tabular}




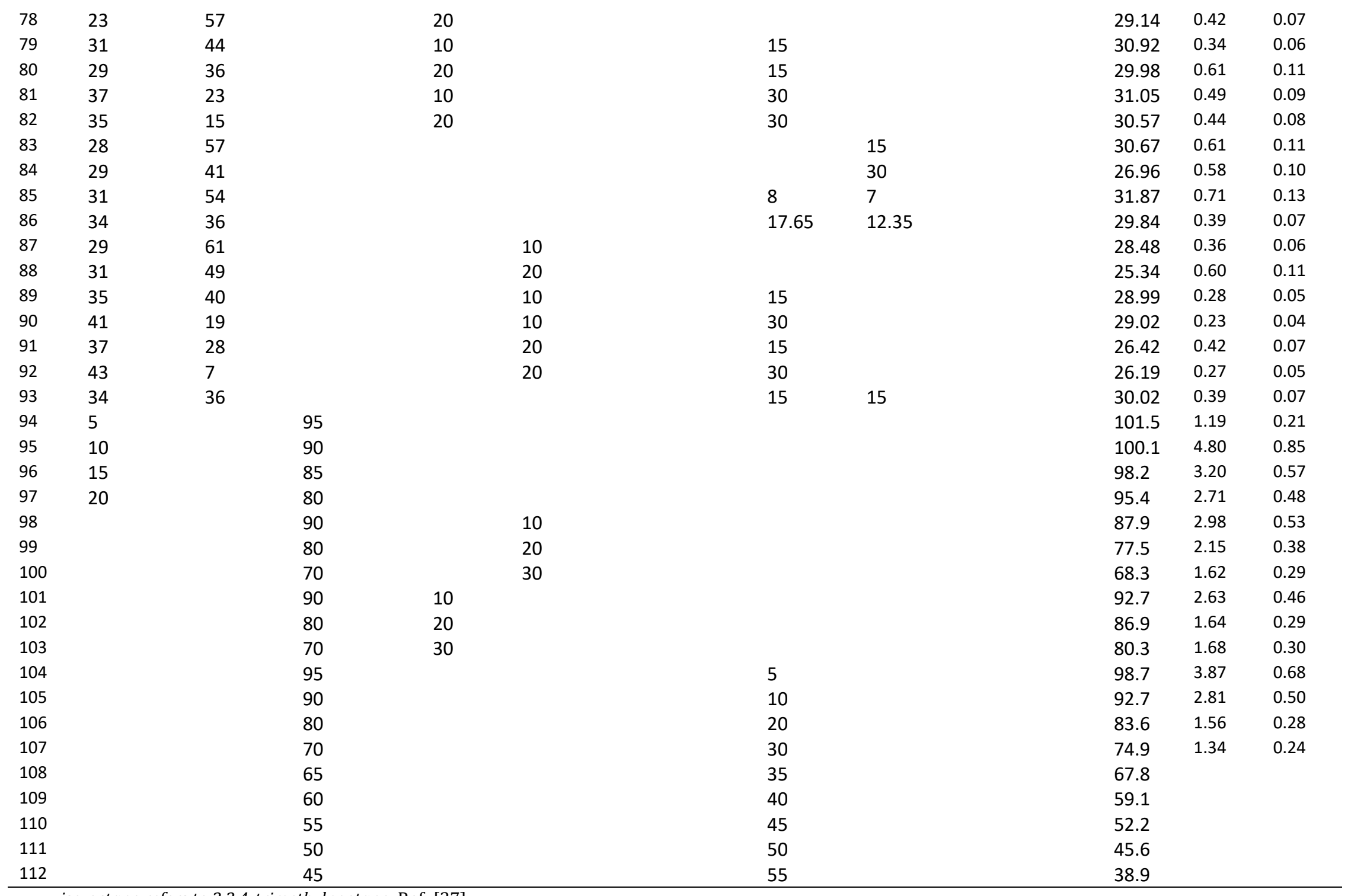

iso-octane refers to 2,2,4-trimethylpentane; Ref: [37] 
Table 3

DCN of blends of pure hydrocarbons and oxygenates

\begin{tabular}{|c|c|c|c|c|c|c|c|c|c|c|c|c|c|c|}
\hline \multirow[t]{2}{*}{ S.no } & \multirow[t]{2}{*}{ Name } & \multicolumn{9}{|c|}{ Components (vol \%) } & \multirow[t]{2}{*}{$\mathrm{DCN}$} & \multirow[t]{2}{*}{ SD } & \multirow[t]{2}{*}{ SE } & \multirow[t]{2}{*}{ Ref } \\
\hline & & $\begin{array}{l}\mathrm{n} \text { - } \\
\text { heptane }\end{array}$ & $\begin{array}{l}\text { iso- } \\
\text { octane }\end{array}$ & toluene & methanol & ethanol & $\begin{array}{l}\mathrm{n}- \\
\text { propanol }\end{array}$ & $\begin{array}{l}\mathrm{n}- \\
\text { butanol }\end{array}$ & $\begin{array}{l}\text { 2-methyl- } \\
\text { butan-1- } \\
\text { ol }\end{array}$ & DEE & & & & \\
\hline 1 & PRF $84+\mathrm{MeOH} 1$ & 15.8 & 82.7 & & 1.5 & & & & & & 25 & 0.54 & 0.10 & [94] \\
\hline 2 & PRF $84+\mathrm{MeOH} 2$ & 15.4 & 81.1 & & 3.5 & & & & & & 23 & 0.42 & 0.07 & [94] \\
\hline 3 & PRF $84+\mathrm{MeOH} 3$ & 14.9 & 78.1 & & 7 & & & & & & 19 & 0.48 & 0.08 & [94] \\
\hline 4 & PRF 70 + EtOH 1 & 29.4 & 68.6 & & & 2 & & & & & 31.62 & 0.41 & 0.07 & [95] \\
\hline 5 & PRF $70+$ EtOH 2 & 28.5 & 66.5 & & & 5 & & & & & 29.14 & 0.42 & 0.07 & [95] \\
\hline 6 & PRF $70+$ EtOH 3 & 27 & 63 & & & 10 & & & & & 25.40 & 0.74 & 0.13 & [95] \\
\hline 7 & PRF $70+$ EtOH 4 & 25.5 & 59.5 & & & 15 & & & & & 22.76 & 0.38 & 0.07 & [95] \\
\hline 8 & PRF $70+$ EtOH 5 & 24 & 56 & & & 20 & & & & & 19.82 & 0.47 & 0.08 & [95] \\
\hline 9 & PRF 84 + EtOH 1 & 15.68 & 82.32 & & & 2 & & & & & 25.42 & 0.42 & 0.07 & [95] \\
\hline 10 & PRF 84 + EtOH 2 & 15.2 & 79.8 & & & 5 & & & & & 23.36 & 0.48 & 0.08 & [95] \\
\hline 11 & PRF $84+$ EtOH 3 & 14.4 & 75.6 & & & 10 & & & & & 20.55 & 0.41 & 0.07 & [95] \\
\hline 12 & PRF $84+$ EtOH 4 & 13.6 & 71.4 & & & 15 & & & & & 17.98 & 0.52 & 0.09 & [95] \\
\hline 13 & PRF 84 + EtOH 5 & 12.8 & 67.2 & & & 20 & & & & & 15.40 & 0.25 & 0.04 & [95] \\
\hline 14 & PRF $70+\mathrm{MeOH}+\mathrm{EtOH} 1$ & 29.4 & 68.7 & & 0.5 & 1.4 & & & & & 32.65 & 0.58 & 0.10 & [94] \\
\hline 15 & PRF $70+\mathrm{MeOH}+\mathrm{EtOH} 2$ & 28.8 & 67 & & 1.7 & 2.5 & & & & & 30.14 & 0.58 & 0.10 & [94] \\
\hline 16 & $\mathrm{PRF} 70+\mathrm{MeOH}+\mathrm{EtOH} 3$ & 27.5 & 64 & & 3.5 & 5 & & & & & 26.55 & 0.43 & 0.08 & [94] \\
\hline 17 & PRF $70+\mathrm{MeOH}+$ EtOH 4 & 26 & 60.8 & & 3.7 & 9.5 & & & & & 23.61 & 0.49 & 0.09 & [94] \\
\hline 18 & PRF $70+\mathrm{MeOH}+\mathrm{EtOH} 5$ & 24.8 & 57.7 & & 5.5 & 12 & & & & & 20.93 & 0.17 & 0.03 & [94] \\
\hline 19 & PRF $70+\mathrm{MeOH}+\mathrm{EtOH} 6$ & 29.5 & 69 & & 1 & 0.5 & & & & & 31.86 & 0.27 & 0.05 & [94] \\
\hline 20 & $\mathrm{PRF} 70+\mathrm{MeOH}+\mathrm{EtOH} 7$ & 28.5 & 68 & & 2.5 & 1.5 & & & & & 30.35 & 0.3 & 0.05 & [94] \\
\hline 21 & PRF $70+\mathrm{MeOH}+\mathrm{EtOH} 8$ & 27 & 66 & & 5 & 3 & & & & & 26.39 & 0.15 & 0.03 & [94] \\
\hline 21 & PRF $70+\mathrm{MeOH}+$ EtOH 9 & 25.5 & 64 & & 7.5 & 4 & & & & & 24.48 & 0.42 & 0.07 & [94] \\
\hline 23 & PRF $70+\mathrm{MeOH}+\mathrm{EtOH} 10$ & 24 & 62 & & 10 & 6 & & & & & 21.75 & 0.24 & 0.04 & [94] \\
\hline 24 & $\mathrm{PRF} 84+\mathrm{MeOH}+\mathrm{EtOH} 1$ & 15.7 & 82.4 & & 0.5 & 1.4 & & & & & 25.51 & 0.39 & 0.07 & [94] \\
\hline 25 & $\mathrm{PRF} 84+\mathrm{MeOH}+\mathrm{EtOH} 2$ & 15.3 & 80.5 & & 1.7 & 2.5 & & & & & 23.48 & 0.33 & 0.06 & [94] \\
\hline 26 & $\mathrm{PRF} 84+\mathrm{MeOH}+\mathrm{EtOH} 3$ & 14.6 & 76.9 & & 3.5 & 5 & & & & & 20.44 & 0.26 & 0.05 & [94] \\
\hline 27 & PRF $84+\mathrm{MeOH}+\mathrm{EtOH} 4$ & 13.9 & 72.9 & & 3.7 & 9.5 & & & & & 18.43 & 0.35 & 0.06 & [94] \\
\hline 28 & PRF $84+\mathrm{MeOH}+\mathrm{EtOH} 5$ & 13.2 & 69.3 & & 5.5 & 12 & & & & & 16.42 & 0.37 & 0.07 & [94] \\
\hline 29 & PRF $84+\mathrm{MeOH}+\mathrm{EtOH} 6$ & 15.68 & 82.82 & & 1 & 0.5 & & & & & 26.34 & 0.31 & 0.05 & [94] \\
\hline 30 & $\mathrm{PRF} 84+\mathrm{MeOH}+\mathrm{EtOH} 7$ & 15.2 & 81.3 & & 2.5 & 1.5 & & & & & 23.76 & 0.46 & 0.08 & [94] \\
\hline 31 & $\mathrm{PRF} 84+\mathrm{MeOH}+\mathrm{EtOH} 8$ & 14.4 & 78.6 & & 5 & 3 & & & & & 20.24 & 0.74 & 0.13 & [94] \\
\hline 32 & PRF 84 + $\mathrm{MeOH}+$ EtOH 9 & 13.6 & 75.9 & & 7.5 & 4 & & & & & 18.47 & 0.2 & 0.04 & [94] \\
\hline 33 & $\mathrm{PRF} 84+\mathrm{MeOH}+\mathrm{EtOH} 10$ & 12.8 & 73.2 & & 10 & 6 & & & & & 15.86 & 0.22 & 0.04 & [94] \\
\hline 34 & PRF $60+$ PrOH 1 & 38 & 57 & & & & 5 & & & & 33.5 & 0.42 & 0.07 & [40] \\
\hline 35 & PRF $60+$ PrOH 2 & 36 & 54 & & & & 10 & & & & 30.1 & 0.53 & 0.09 & [40] \\
\hline 36 & PRF $60+$ PrOH 3 & 34 & 51 & & & & 15 & & & & 25 & 0.42 & 0.07 & [40] \\
\hline 37 & PRF $60+\operatorname{PrOH} 4$ & 32 & 48 & & & & 20 & & & & 20.4 & 0.28 & 0.05 & [40] \\
\hline 38 & PRF $60+$ PrOH 5 & 28 & 42 & & & & 30 & & & & 16.2 & 0.34 & 0.06 & [40] \\
\hline 39 & PRF $60+$ PrOH 6 & 20 & 30 & & & & 50 & & & & 13.7 & 0.19 & 0.03 & [40] \\
\hline 40 & PRF 60 + PrOH 7 & 12 & 18 & & & & 70 & & & & 9.2 & 0.19 & 0.03 & [40] \\
\hline
\end{tabular}




\begin{tabular}{|c|c|c|c|}
\hline PRF 70 + PrOH 1 & 28.5 & 66.5 & \\
\hline PRF 70 + PrOH 2 & 27 & 63 & \\
\hline PRF 70 + PrOH 3 & 25.5 & 59.5 & \\
\hline PRF $70+\mathrm{PrOH} 4$ & 24 & 56 & \\
\hline PRF $70+$ PrOH 5 & 21 & 49 & \\
\hline PRF $70+$ PrOH 6 & 15 & 35 & \\
\hline PRF 70 + PrOH 7 & 9 & 21 & \\
\hline PRF 84 + PrOH 1 & 15.2 & 79.8 & \\
\hline PRF 84 + PrOH 2 & 14.4 & 75.6 & \\
\hline PRF 84 + $\mathrm{PrOH} 3$ & 13.6 & 71.4 & \\
\hline PRF $84+\mathrm{PrOH} 4$ & 12.8 & 67.2 & \\
\hline PRF $84+$ PrOH 5 & 11.2 & 58.8 & \\
\hline PRF 84 + PrOH 6 & 8 & 42 & \\
\hline PRF 84 + PrOH 7 & 4.8 & 25.2 & \\
\hline PRF $60+\mathrm{BuOH} 1$ & 38 & 57 & \\
\hline PRF $60+\mathrm{BuOH} 2$ & 36 & 54 & \\
\hline PRF $60+\mathrm{BuOH} 3$ & 32 & 48 & \\
\hline PRF $60+\mathrm{BuOH} 4$ & 28 & 42 & \\
\hline PRF $60+$ BuOH 5 & 20 & 30 & \\
\hline PRF $60+\mathrm{BuOH} 6$ & 12 & 18 & \\
\hline PRF $70+$ BuOH 1 & 28.5 & 66.5 & \\
\hline PRF $70+\mathrm{BuOH} 2$ & 27 & 63 & \\
\hline PRF $70+\mathrm{BuOH} 3$ & 25.5 & 59.5 & \\
\hline PRF $70+\mathrm{BuOH} 4$ & 24 & 56 & \\
\hline PRF $70+$ BuOH 5 & 21 & 49 & \\
\hline PRF $70+$ BuOH 6 & 15 & 35 & \\
\hline PRF $70+$ BuOH 7 & 9 & 21 & \\
\hline PRF 84 + BuOH 1 & 15.2 & 79.8 & \\
\hline PRF $84+$ BuOH 2 & 14.4 & 75.6 & \\
\hline PRF $84+\mathrm{BuOH} 3$ & 13.6 & 71.4 & \\
\hline PRF $84+\mathrm{BuOH} 4$ & 12.8 & 67.2 & \\
\hline PRF $84+\mathrm{BuOH} 5$ & 11.2 & 58.8 & \\
\hline PRF $84+\mathrm{BuOH} 6$ & 8 & 42 & \\
\hline PRF 84 + BuOH 7 & 4.8 & 25.2 & \\
\hline PRF 70 + MBuOH 1 & 28.5 & 66.5 & \\
\hline PRF $70+\mathrm{MBuOH} 2$ & 27 & 63 & \\
\hline PRF $70+\mathrm{MBuOH} 3$ & 24 & 56 & \\
\hline PRF $84+\mathrm{MBuOH} 1$ & 15.2 & 79.8 & \\
\hline PRF 84 + MBuOH 2 & 14.4 & 75.6 & \\
\hline PRF 84 + MBuOH 2 & 12.8 & 67.2 & \\
\hline TPRF + BuOH 1 & 35.15 & 36.1 & 23.75 \\
\hline TPRF + BuOH 2 & 33.3 & 34.2 & 22.5 \\
\hline TPRF + BuOH 3 & 29.6 & 30.4 & 20 \\
\hline TPRF + MBuOH 1 & 35.15 & 36.1 & 23.75 \\
\hline TPRF + MBuOH 2 & 33.3 & 34.2 & 22.5 \\
\hline TPRF + MBuOH 3 & 29.6 & 30.4 & 20 \\
\hline n-heptane + DEE 25 & 75 & 0 & \\
\hline
\end{tabular}

$\begin{array}{llll}28.8 & 0.3 & 0.05 & {[40]} \\ 26.1 & 0.41 & 0.07 & {[40]} \\ 24.34 & 0.47 & 0.08 & {[40]} \\ 21.7 & 0.4 & 0.07 & {[40]} \\ 17.8 & 0.38 & 0.07 & {[40]} \\ 11.85 & 0.1 & 0.02 & {[40]} \\ 8.49 & 0.3 & 0.05 & {[40]} \\ 23.5 & 0.42 & 0.07 & {[40]} \\ 21.4 & 0.22 & 0.04 & {[40]} \\ 18.91 & 0.38 & 0.07 & {[40]} \\ 17.3 & 0.31 & 0.05 & {[40]} \\ 13.7 & 0.23 & 0.04 & {[40]} \\ 9.27 & 0.2 & 0.04 & {[40]} \\ 8.4 & 0.2 & 0.04 & {[40]} \\ 33.8 & 1 & 0.18 & {[40]} \\ 32 & 0.7 & 0.12 & {[40]} \\ 28.4 & 0.54 & 0.10 & {[40]} \\ 24.9 & 0.4 & 0.07 & {[40]} \\ 19.4 & 0.2 & 0.04 & {[40]} \\ 15.1 & 0.2 & 0.04 & {[40]} \\ 29.5 & 0.44 & 0.08 & {[40]} \\ 27.21 & 0.25 & 0.04 & {[40]} \\ 26.55 & 0.42 & 0.07 & {[40]} \\ 24.5 & 0.54 & 0.10 & {[40]} \\ 21.3 & 0.5 & 0.09 & {[40]} \\ 17.1 & 0.3 & 0.05 & {[40]} \\ 14 & 0.2 & 0.04 & {[40]} \\ 23.9 & 0.38 & 0.07 & {[40]} \\ 22.81 & 0.36 & 0.06 & {[40]} \\ 21.25 & 0.44 & 0.08 & {[40]} \\ 20.21 & 0.57 & 0.10 & {[40]} \\ 17.96 & 0.4 & 0.07 & {[40]} \\ 15.17 & 0.2 & 0.04 & {[40]} \\ 13.09 & 0.1 & 0.02 & {[40]} \\ 29.2 & 0.47 & 0.08 & \text { measured } \\ 28.1 & 0.36 & 0.06 & \text { measured } \\ 24.9 & 0.45 & 0.08 & \text { measured } \\ 23.6 & 0.72 & 0.13 & \text { measured } \\ 21.9 & 0.44 & 0.08 & \text { measured } \\ 18.9 & 0.28 & 0.05 & \text { measured } \\ 30.4 & 0.4 & 0.07 & \text { measured } \\ 28.8 & 0.29 & 0.05 & \text { measured } \\ 25.7 & 0.18 & 0.03 & \text { measured } \\ 29.5 & 0.22 & 0.04 & \text { measured } \\ 27.7 & 0.31 & 0.05 & \text { measured } \\ 25.4 & 0.51 & 0.09 & \text { measured } \\ 62.2 & 1.13 & 0.20 & \text { measured }\end{array}$




\begin{tabular}{|c|c|c|c|c|c|c|c|c|c|c|}
\hline n-heptane + DEE 50 & 50 & 0 & & & & 50 & 79.4 & 1.49 & 0.26 & measured \\
\hline n-heptane + DEE 75 & 25 & 0 & & & & 75 & 106.2 & 3.78 & 0.67 & measured \\
\hline iso-octane + DEE 50 & 0 & 50 & & & & 50 & 66.6 & 1.55 & 0.27 & measured \\
\hline iso-octane + DEE 75 & 0 & 25 & & & & 75 & 93.4 & 3.57 & 0.63 & measured \\
\hline iso-octane + DEE 25 & 0 & 75 & & & & 25 & 44.3 & 1.17 & 0.21 & measured \\
\hline $\mathrm{MeOH}+\mathrm{DEE} 40$ & & & 60 & & & 40 & 11.1 & 0.78 & 0.14 & measured \\
\hline $\mathrm{MeOH}+\mathrm{DEE} 50$ & & & 50 & & & 50 & 20.2 & 0.88 & 0.16 & measured \\
\hline $\mathrm{MeOH}+\mathrm{DEE} 80$ & & & 20 & & & 80 & 66.1 & 1.42 & 0.25 & measured \\
\hline $\mathrm{MeOH}+\mathrm{DEE} 90$ & & & 10 & & & 90 & 97.2 & 2.45 & 0.43 & measured \\
\hline EtOH + DEE 10 & & & & 90 & & 10 & 7.8 & 0.21 & 0.04 & measured \\
\hline EtOH + DEE 20 & & & & 80 & & 20 & 8.2 & 0.16 & 0.03 & measured \\
\hline EtOH + DEE 25 & & & & 75 & & 25 & 9.2 & 0.19 & 0.03 & measured \\
\hline EtOH + DEE 30 & & & & 70 & & 30 & 10.5 & 0.3 & 0.05 & measured \\
\hline EtOH + DEE 35 & & & & 65 & & 35 & 11 & 0.24 & 0.04 & measured \\
\hline EtOH + DEE 40 & & & & 60 & & 40 & 16.9 & 0.34 & 0.06 & measured \\
\hline EtOH + DEE 50 & & & & 50 & & 50 & 23.8 & 0.32 & 0.06 & measured \\
\hline EtOH + DEE 60 & & & & 40 & & 60 & 35.5 & 0.58 & 0.10 & measured \\
\hline EtOH + DEE 70 & & & & 30 & & 70 & 48.9 & 0.85 & 0.15 & measured \\
\hline EtOH + DEE 75 & & & & 25 & & 75 & 56.6 & 0.74 & 0.13 & measured \\
\hline EtOH + DEE 80 & & & & 20 & & 80 & 67.9 & 1.27 & 0.22 & measured \\
\hline EtOH + DEE 90 & & & & 10 & & 90 & 97.7 & 2.95 & 0.52 & measured \\
\hline $\mathrm{BuOH}+\mathrm{DEE} 90$ & & & & & 10 & 90 & 101.9 & 2.42 & 0.43 & measured \\
\hline $\mathrm{BuOH}+\mathrm{DEE} 80$ & & & & & 20 & 80 & 71.9 & 2.01 & 0.36 & measured \\
\hline
\end{tabular}

Expansion of the notations. MeOH: methanol; EtOH: ethanol; PrOH: n-propanol; BuOH: $n$-butanol; MBuOH: 2-methyl-butan-1-ol and DEE: diethyl

ether; 
Table 4

DCN of blends of real fuels and oxygenates

\begin{tabular}{|c|c|c|c|c|c|c|c|c|c|c|c|c|c|c|c|c|c|c|c|c|}
\hline \multirow[t]{2}{*}{ S.no } & \multirow[t]{2}{*}{ Name } & \multicolumn{15}{|c|}{ Components (vol \%) } & \multirow[t]{2}{*}{$\mathrm{DCN}$} & \multirow[t]{2}{*}{ SD } & \multirow[t]{2}{*}{ SE } & \multirow[t]{2}{*}{ Ref } \\
\hline & & $\begin{array}{l}\text { FACE } \\
\text { A }\end{array}$ & $\begin{array}{l}\text { FACE } \\
1\end{array}$ & $\begin{array}{l}\text { FACE } \\
\mathrm{J}\end{array}$ & SG & $\begin{array}{l}\text { LN } \\
\mathrm{J} 80\end{array}$ & $\begin{array}{l}\mathrm{HN} \\
\mathrm{J} 80\end{array}$ & $\mathrm{MeOH}$ & $\mathrm{EtOH}$ & $\mathrm{PrOH}$ & $\mathrm{BuOH}$ & DEE & DPE & DIPE & DBE & DMM & & & & \\
\hline 1 & FACE A & 100 & & & & & & & & & & & & & & & 26.4 & 0.60 & 0.11 & [9] \\
\hline 2 & FACE I & & 100 & & & & & & & & & & & & & & 31.8 & 0.40 & 0.07 & [9] \\
\hline 3 & FACE J & & & 100 & & & & & & & & & & & & & 29.7 & 0.70 & 0.12 & [9] \\
\hline 4 & SG & & & & 100 & & & & & & & & & & & & 19.2 & 0.27 & 0.05 & [96] \\
\hline 5 & LN J80 & & & & & 100 & & & & & & & & & & & 27.6 & 0.57 & 0.10 & [96] \\
\hline 6 & HN J80 & & & & & & 100 & & & & & & & & & & 31 & 0.39 & 0.07 & [96] \\
\hline 7 & FACE A + MeOH 1.5 & 98.5 & & & & & & 1.5 & & & & & & & & & 25.7 & 0.20 & 0.04 & [94] \\
\hline 9 & FACE A + MeOH 7 & 93 & & & & & & 7 & & & & & & & & & 21 & 0.30 & 0.05 & [94] \\
\hline 10 & FACE I + MeOH 1.5 & & 98.5 & & & & & 1.5 & & & & & & & & & 30.6 & 0.30 & 0.05 & [94] \\
\hline 11 & FACE I + MeOH 3.5 & & 96.5 & & & & & 3.5 & & & & & & & & & 29.5 & 0.30 & 0.05 & [94] \\
\hline 12 & FACE J + MeOH 1.5 & & & 98.5 & & & & 1.5 & & & & & & & & & 29.2 & 0.30 & 0.05 & [94] \\
\hline 13 & FACE J + MeOH 3.5 & & & 96.5 & & & & 3.5 & & & & & & & & & 27.9 & 0.30 & 0.05 & [94] \\
\hline 14 & $\mathrm{FACE} \mathrm{J}+\mathrm{MeOH} 7$ & & & 93 & & & & 7 & & & & & & & & & 26.1 & 0.30 & 0.05 & [94] \\
\hline 15 & FACE J + MeOH 10.5 & & & 89.5 & & & & 10.5 & & & & & & & & & 25 & 0.60 & 0.11 & [94] \\
\hline 16 & FACE J + MeOH 14 & & & 86 & & & & 14 & & & & & & & & & 22.9 & 0.20 & 0.04 & [94] \\
\hline 17 & FACE A + EtOH 2 & 98 & & & & & & & 2 & & & & & & & & 25.18 & 0.42 & 0.07 & [95] \\
\hline 18 & FACE A + EtOH 5 & 95 & & & & & & & 5 & & & & & & & & 23.57 & 0.25 & 0.04 & [95] \\
\hline 20 & FACE A + EtOH 15 & 85 & & & & & & & 15 & & & & & & & & 18.53 & 0.43 & 0.08 & [95] \\
\hline 21 & FACE A + EtOH 20 & 80 & & & & & & & 20 & & & & & & & & 16.11 & 0.49 & 0.09 & [95] \\
\hline 21 & FACE I + EtOH 2 & & 98 & & & & & & 2 & & & & & & & & 30.65 & 0.49 & 0.09 & [95] \\
\hline 23 & FACE I + EtOH 5 & & 95 & & & & & & 5 & & & & & & & & 28.92 & 0.41 & 0.07 & [95] \\
\hline 24 & FACE I + EtOH 10 & & 90 & & & & & & 10 & & & & & & & & 26.10 & 0.52 & 0.09 & [95] \\
\hline 25 & FACE I + EtOH 15 & & 85 & & & & & & 15 & & & & & & & & 23.32 & 0.32 & 0.06 & [95] \\
\hline 26 & FACE I + EtOH 20 & & 80 & & & & & & 20 & & & & & & & & 20.73 & 0.41 & 0.07 & [95] \\
\hline 27 & FACE J + EtOH 2 & & & 98 & & & & & 2 & & & & & & & & 28.49 & 0.24 & 0.04 & [95] \\
\hline 28 & FACE J + EtOH 5 & & & 95 & & & & & 5 & & & & & & & & 26.85 & 0.74 & 0.13 & [95] \\
\hline 29 & FACE J + EtOH 10 & & & 90 & & & & & 10 & & & & & & & & 24.45 & 0.64 & 0.11 & [95] \\
\hline 30 & FACE J + EtOH 15 & & & 85 & & & & & 15 & & & & & & & & 22.50 & 0.68 & 0.12 & [95] \\
\hline 31 & FACE J + EtOH 20 & & & 80 & & & & & 20 & & & & & & & & 20.93 & 0.36 & 0.06 & [95] \\
\hline 32 & $\mathrm{FACE} \mathrm{A}+\mathrm{MeOH}+\mathrm{EtOH} 1$ & 98.1 & & & & & & 0.5 & 1.4 & & & & & & & & 25.71 & 0.18 & 0.03 & [94] \\
\hline 33 & $\mathrm{FACE} A+\mathrm{MeOH}+\mathrm{EtOH} 2$ & 95.8 & & & & & & 1.7 & 2.5 & & & & & & & & 23.82 & 0.30 & 0.05 & [94] \\
\hline 34 & $\mathrm{FACE} \mathrm{A}+\mathrm{MeOH}+\mathrm{EtOH} 3$ & 91.5 & & & & & & 3.5 & 5 & & & & & & & & 21.76 & 0.46 & 0.08 & [94] \\
\hline 35 & $\mathrm{FACE} \mathrm{A}+\mathrm{MeOH}+\mathrm{EtOH} 4$ & 86.8 & & & & & & 3.7 & 9.5 & & & & & & & & 19.21 & 0.16 & 0.03 & [94] \\
\hline 36 & $\mathrm{FACE} \mathrm{A}+\mathrm{MeOH}+\mathrm{EtOH} 5$ & 82.5 & & & & & & 5.5 & 12 & & & & & & & & 17.27 & 0.26 & 0.05 & [94] \\
\hline 37 & $\mathrm{FACE} A+\mathrm{MeOH}+\mathrm{EtOH} 6$ & 98.5 & & & & & & 1 & 0.5 & & & & & & & & 25.46 & 0.22 & 0.04 & [94] \\
\hline 38 & $\mathrm{FACE} A+\mathrm{MeOH}+\mathrm{EtOH} 7$ & 96 & & & & & & 2.5 & 1.5 & & & & & & & & 23.89 & 0.37 & 0.07 & [94] \\
\hline
\end{tabular}


$\mathrm{FACE} I+\mathrm{MeOH}+\mathrm{EtOH} 1$

$\mathrm{FACE} I+\mathrm{MeOH}+\mathrm{EtOH} 2$

$\mathrm{FACE} \mathrm{I}+\mathrm{MeOH}+\mathrm{EtOH} 3$

$\mathrm{FACE} I+\mathrm{MeOH}+\mathrm{EtOH} 4$

$\mathrm{FACE} I+\mathrm{MeOH}+\mathrm{EtOH} 5$

$\mathrm{FACE} I+\mathrm{MeOH}+\mathrm{EtOH} 6$

$\mathrm{FACE} \mathrm{I}+\mathrm{MeOH}+\mathrm{EtOH} 7$

$\mathrm{FACE} \mathrm{I}+\mathrm{MeOH}+\mathrm{EtOH} 8$

$\mathrm{FACE} I+\mathrm{MeOH}+\mathrm{EtOH} 9$

$\mathrm{FACE} \mathrm{I}+\mathrm{MeOH}+\mathrm{EtOH} 10$

FACE J + $\mathrm{MeOH}+\mathrm{EtOH} 1$

$\mathrm{FACE} \mathrm{J}+\mathrm{MeOH}+\mathrm{EtOH} 2$

FACE J + MeOH + EtOH 3

$\mathrm{FACE} \mathrm{J}+\mathrm{MeOH}+\mathrm{EtOH} 4$

$\mathrm{FACE} \mathrm{J} \mathrm{+} \mathrm{MeOH}+\mathrm{EtOH} 5$

$\mathrm{FACE} \mathrm{J}+\mathrm{MeOH}+\mathrm{EtOH} 6$

$\mathrm{FACE} \mathrm{J}+\mathrm{MeOH}+\mathrm{EtOH} 7$

$\mathrm{FACE} \mathrm{J}+\mathrm{MeOH}+\mathrm{EtOH} 8$

$\mathrm{FACE} \mathrm{J}+\mathrm{MeOH}+\mathrm{EtOH} 9$

FACE A + PrOH 5

FACE A + PrOH 10

FACE A + PrOH 15

FACE A + PrOH 20

FACE A + PrOH 30

FACE A + PrOH 50

FACE A + PrOH 70

FACE I + PrOH 5

FACE I + PrOH 10

FACE I + PrOH 15

FACE I + PrOH 20

FACE I + PrOH 30

FACE I + PrOH 50

FACE I + PrOH 70

FACE J + PrOH 5

FACE J + PrOH 10

FACE J + PrOH 15

FACE J + PrOH 20

FACE J + PrOH 30

FACE J + PrOH 50

FACE J + PrOH 70

FACE $\mathrm{A}+\mathrm{BuOH} 5$

FACE A + BuOH 10

FACE A + BuOH 15

FACE A + BuOH 20

FACE $\mathrm{A}+\mathrm{BUOH} 30$

FACE A + BuOH 50

FACE A + BuOH 70
98.1

95.8

91.5

86.8

82.5

98.5

96

92
88.5

95.8

91.5

86.8

82.5

98.5

96

92

88.5

95

85

80
70

30

95

90

70
50

30

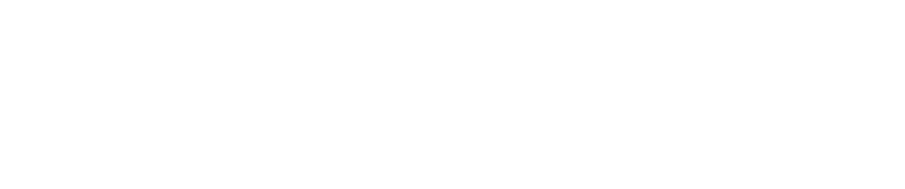

5
10
15
20
30
50
70
5
10
15
20
30
50
70
5
10
15
20
30
50
70

9.5

0.5

4

1.4

5

9.5

12

0.5

5
10
15
20
30
50
70

$\begin{array}{llll}30.91 & 0.32 & 0.06 & {[94]} \\ 28.97 & 0.28 & 0.05 & {[94]} \\ 26.04 & 0.45 & 0.08 & {[94]} \\ 23.53 & 0.19 & 0.03 & {[94]} \\ 21.44 & 0.55 & 0.10 & {[94]} \\ 30.7 & 0.34 & 0.06 & {[94]} \\ 29.27 & 0.29 & 0.05 & {[94]} \\ 26.65 & 0.23 & 0.04 & {[94]} \\ 24.6 & 0.41 & 0.07 & {[94]} \\ 22.1 & 0.22 & 0.04 & {[94]} \\ 28.38 & 0.31 & 0.05 & {[94]} \\ 27.18 & 0.3 & 0.05 & {[94]} \\ 25.28 & 0.34 & 0.06 & {[94]} \\ 22.92 & 0.35 & 0.06 & {[94]} \\ 21.11 & 0.22 & 0.04 & {[94]} \\ 29.06 & 0.4 & 0.07 & {[94]} \\ 27.44 & 0.35 & 0.06 & {[94]} \\ 25.84 & 0.19 & 0.03 & {[94]} \\ 24.17 & 0.41 & 0.07 & {[94]} \\ 23.8 & 0.35 & 0.06 & {[40]} \\ 21.8 & 0.59 & 0.10 & {[40]} \\ 19.6 & 0.43 & 0.08 & {[40]} \\ 18.2 & 0.23 & 0.04 & {[40]} \\ 14.9 & 0.35 & 0.06 & {[40]} \\ 10.04 & 0.17 & 0.03 & {[40]} \\ 8.6 & 0.09 & 0.02 & {[40]} \\ 29.9 & 0.48 & 0.08 & {[40]} \\ 28 & 0.43 & 0.08 & {[40]} \\ 26.8 & 0.39 & 0.07 & {[40]} \\ 25 & 0.27 & 0.05 & {[40]} \\ 21.7 & 0.30 & 0.05 & {[40]} \\ 17.9 & 0.30 & 0.05 & {[40]} \\ 14.5 & 0.20 & 0.04 & {[40]} \\ 25.92 & 0.44 & 0.08 & {[40]} \\ 24.2 & 0.29 & 0.05 & {[40]} \\ 22.6 & 0.39 & 0.07 & {[40]} \\ 21.27 & 0.31 & 0.05 & {[40]} \\ 18.46 & 0.12 & 0.02 & {[40]} \\ 12.9 & 0.24 & 0.04 & {[40]} \\ 8.9 & 0.16 & 0.03 & {[40]} \\ 24.1 & 0.35 & 0.06 & {[40]} \\ 23.4 & 0.59 & 0.10 & {[40]} \\ 21.36 & 0.47 & 0.08 & {[40]} \\ 20.45 & 0.23 & 0.04 & {[40]} \\ 18.92 & 0.35 & 0.06 & {[40]} \\ 15.61 & 0.17 & 0.03 & {[40]} \\ 13.18 & 0.09 & 0.02 & {[40]}\end{array}$


$\mathrm{FACE} I+\mathrm{BuOH} 5$

FACE I + BuOH 10

FACE I + BuOH 15

FACE I + BuOH 20

FACE I + BuOH 30

FACE I + BuOH 50

$\mathrm{FACE} I+\mathrm{BuOH} 70$

FACE J + BuOH 5

FACE J + BuOH 10

FACE J + BuOH 15

FACE J + BuOH 20

FACE J + BuOH 30

$\mathrm{FACE} \mathrm{J} \mathrm{+} \mathrm{BuOH} 50$

FACE J + BuOH 70

$S G+D E E ~ 1$

$S G+D E E 5$

SG + DEE 10

SG + DEE 20

SG + DPE 1

SG + DPE 5

$S G+D P E 10$

SG + DPE 20

SG + DIPE 1

SG + DIPE 5

SG + DIPE 10

SG + DIPE 20

SG + DBE 5

SG + DBE 10

$S G+D B E 15$

SG + DBE 20

$S G+D M M 1$

SG + DMM 20

LN J80 + DEE 1

LN J80 + DEE 5

LN J80 + DEE 10

LN J80 + DEE 20

HN J80 + DEE 1

HN J80 + DEE 5

HN J80 + DEE 10

HN J80 + DEE 20

LN J80 + DPE 1

LN J80 + DPE 5

LN J80 + DPE 10

LN J80 + DPE 20

HN J80 + DPE 1

HN J80 + DPE 5

HN J80 + DPE 10 
HN J80 + DPE 20

LN J80 + DIPE 1

LN J80 + DIPE 5

LN J80 + DIPE 10

LN J80 + DIPE 20

HN J80 + DIPE 1

HN J80 + DIPE 5

HN J80 + DIPE 10

HN J80 + DIPE 20

LN J80 + DBE 5

LN J80 + DBE 10

LN J80 + DBE 15

LN J80 + DBE 20

HN J80 + DBE 5

HN J80 + DBE 10

HN J80 + DBE 15

HN J80 + DBE 20

LN J80 + DMM 1

LN J80 + DMM 20

HN J80 + DMM 1

HN J80 + DMM 20

FACE C gasoline

FACE F gasoline

FACE G gasoline

EXpansion of notations. FACE A: FACEA gasoline; FACE I: FACE I gasoline; FACE J: FACE J gasoline; SG; satorp gasoline; LN J80: light naphtha J80; HN J80: heavy naphtha J80; DEE:

diethyl ether; DPE: dipropyl ether; DIPE: diisopropyl ether; DBE:dibutyl ether and DMM:dimethoxymethane;
20

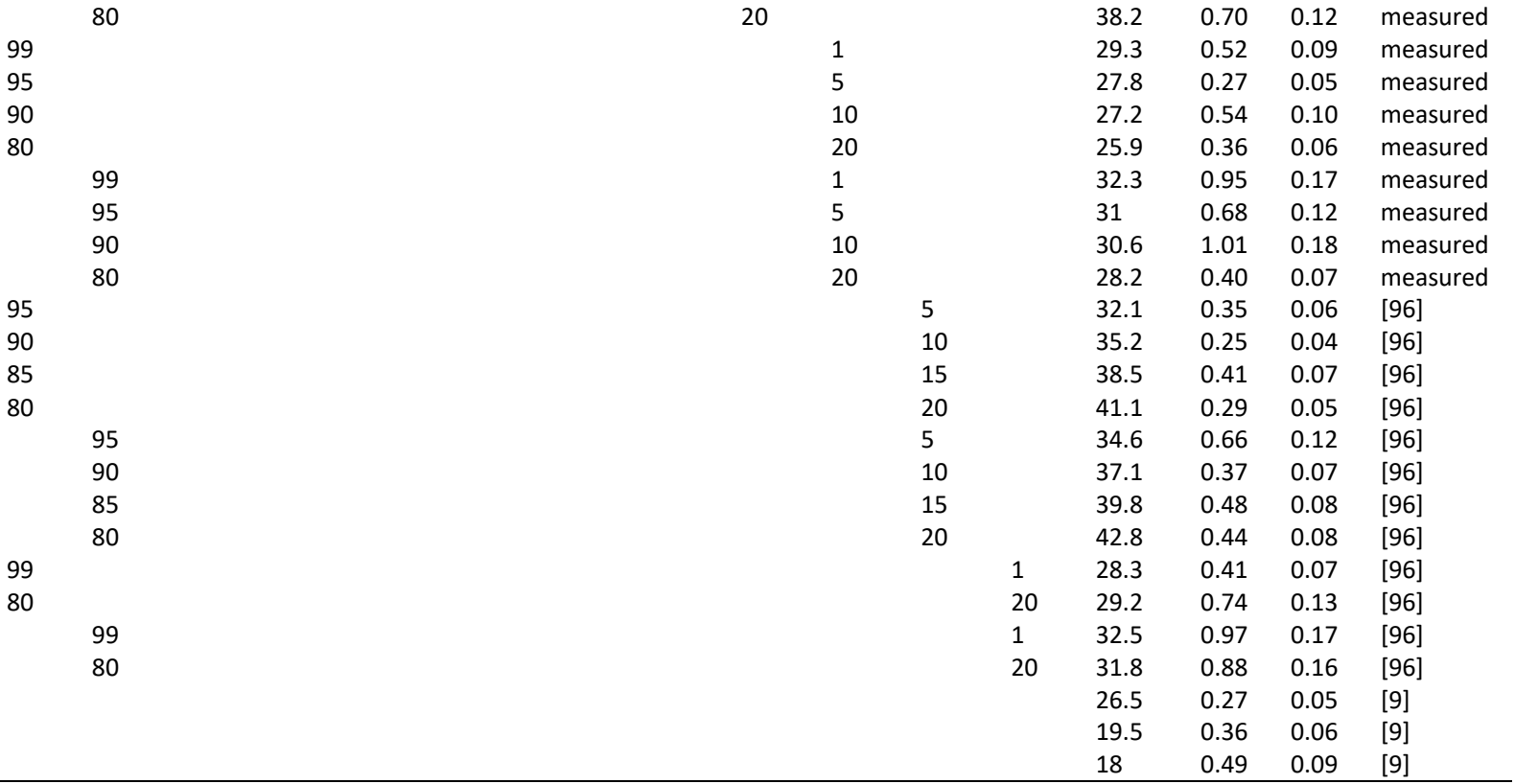




\section{COMPUTATIONAL METHODOLOGIES}

\subsection{Functional group determination}

The functional groups for pure compounds are calculated from their molecular structure. Consider the example of $n$-pentane $(\mathrm{MW}=72 \mathrm{~g} / \mathrm{mol})$; it can be disassembled into two paraffinic $\mathrm{CH}_{3}$ groups ( $\mathrm{MW}=15$ $\mathrm{g} / \mathrm{mol}$ ) and three paraffinic $\mathrm{CH}_{2}$ groups ( $\mathrm{MW}=14 \mathrm{~g} / \mathrm{mol}$ ). The total contribution of paraffinic $\mathrm{CH}_{3}$ groups in $\mathrm{n}$-pentane is $(2 * 15) \mathrm{g} / \mathrm{mol}$ and in terms of weight percent (wt \%) this equates to $41.7 \%$. The remaining 58.3 wt \% is made up of paraffinic $\mathrm{CH}_{2}$ groups. Using a similar analogy, 1-hexene (MW= 84 $\mathrm{g} / \mathrm{mol}$ ) has one paraffinic $\mathrm{CH}_{3}$ group, three paraffinic $\mathrm{CH}_{2}$ groups, one olefinic $-\mathrm{CH}=$ group (MW= 13 $\mathrm{g} / \mathrm{mol}$ ) and one olefinic $=\mathrm{CH}_{2}$ group $(\mathrm{MW}=14 \mathrm{~g} / \mathrm{mol})$. The wt $\%$ of paraffinic $\mathrm{CH}_{3}$ groups, paraffinic $\mathrm{CH}_{2}$ groups and olefinic $-\mathrm{CH}=\mathrm{CH}_{2}$ groups in 1-hexene is $17.9,50.0$ and $32.1 \%$, respectively. The distribution of functional groups present in a few pure compounds is presented in Fig. 1. The functional groups are expressed in wt \% and not as mol \% because the values obtained for the latter might be misleading as observed in our previous study [37]. A paraffinic $\mathrm{CH}_{3}$ group contains 4 atoms (one carbon and three hydrogen atoms) whereas a paraffinic $\mathrm{CH}$ group contains only two atoms (one carbon and one hydrogen atom). Expressing these functional groups in mol \% would result in a value of $50 \%$ each and lead to statistical under representation of the paraffinic $\mathrm{CH}_{3}$ groups (numerically) and over representation of the other. This problem would be severe for other compounds like toluene, methylcyclohexane, etc.

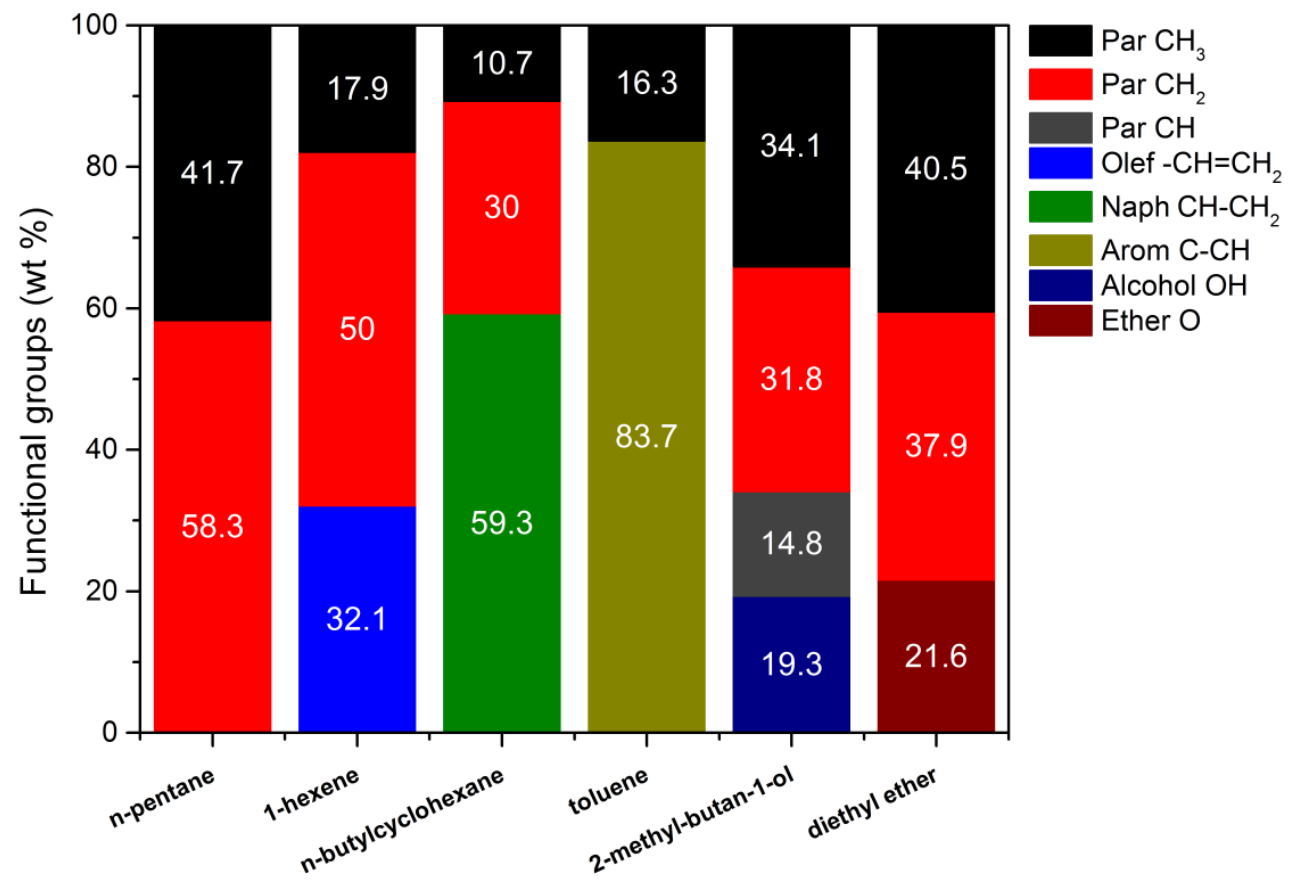

Fig. 1. Distribution of functional groups present in some pure compounds. 
Liquid fuels are often blended in volume fractions and the functional group distribution in such blends can be calculated by converting them into weight fractions (by using pure component densities). Fig. 2. shows the functional groups present in the following blends; PRF 70 (2,2,4-trimethylpentane 70\%, nheptane 30\%); PRF 70 + $25 \%$ 1-hexene (2,2,4-trimethylpentane 38\%, n-heptane 37\%, 1-hexene 25\%); PRF $70+25 \%$ CPT (2,2,4-trimethylpentane 38\%, n-heptane 37\%, cyclopentane 25\%); PRF $70+25 \%$ toluene (2,2,4-trimethylpentane 38\%, n-heptane 37\%, toluene 25\%); PRF $70+25 \%$ ethanol (2,2,4trimethylpentane 38\%, n-heptane 37\%, ethanol 25\%) and PRF $70+25 \%$ DEE (2,2,4-trimethylpentane $38 \%$, n-heptane $37 \%$, diethylether $25 \%$ ). The compositions of the above blends are expressed in vol \%. The functional groups reported in Fig. 2. do not add up to $100 \%$ because quaternary paraffinic carbon atoms present in 2,2,4-trimethylpentane (which make up the remaining \%) are not considered amongst the functional groups in the present study.

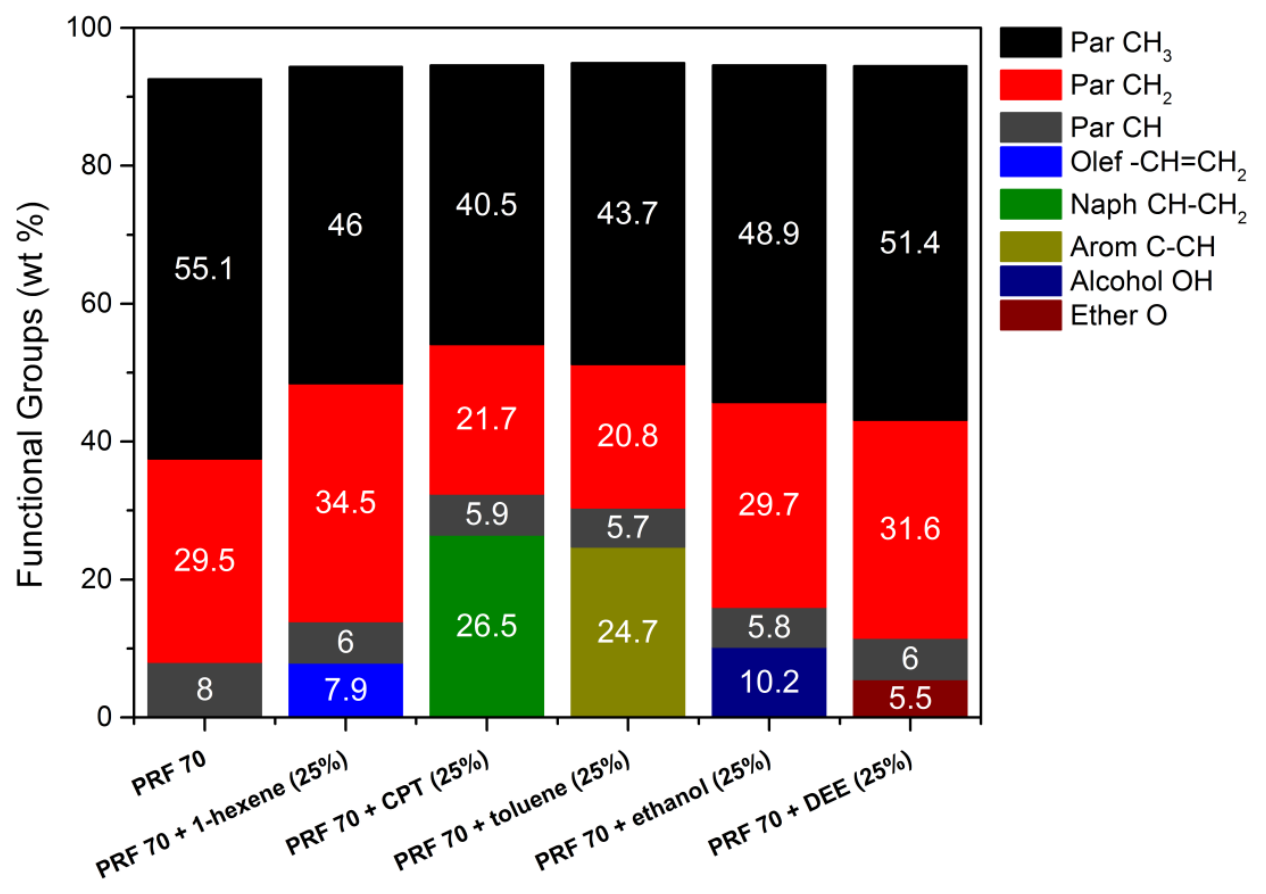

Fig. 2. Distribution of the eight functional groups present in fuel blends.

The hydrocarbon functional groups present in real fuels like gasoline, diesel etc. can be evaluated from ${ }^{1} \mathrm{H}$ NMR spectra. The functional groups present in the fuel generate NMR resonances or peaks at characteristic chemical shifts. One of the important advantages of ${ }^{1} \mathrm{H}$ NMR spectra is that it is a quantitative technique where the composition of each functional group relative to each other can be estimated by comparing the peak areas. The standard chemical shift assignments for each functional group and the detailed procedure to calculate these functional groups (in wt \%) has been presented in 
detail in our previous work [66]. Identifying and quantifying the oxygenated functional groups from ${ }^{1} \mathrm{H}$ NMR spectra alone may not be possible. Oxygenates like alcohols and ethers are usually not present in real petroleum fuels and these are blended in known quantities. Prior knowledge of the oxygenate blend ratio and molecular structure of the oxygenate species is needed for determining the quantity of alcohol $\mathrm{OH}$ and ether $\mathrm{O}$ groups in the fuel.

Molecular weight is an additional input parameter for the model and has a notable influence on the physical properties of a fuel. The molecular weight of pure compounds and their blends are determined from the molecular structure, whereas the molecular weight for real fuels can be predicted using theoretical models or measured using techniques like vapor pressure depression [97], gel permeation chromatography [98], distillation curves [99], etc. BI, another input feature of the model, is a quantity that represents the "extent and quality of branching in a molecule" and was introduced by Abdul Jameel et al. [37]. Branching has a negative effect on DCN [100-103] and positive effect on octane rating [66]. The number of alkyl branches and the position of these branches together determine the reactivity of any molecule. Let us consider 2-methylheptane which has a DCN of 52.6 and 3methylheptane which has a DCN of 45. Both these compounds have the same degree of branching (and functional group distribution) but their DCNs differ by more than $10 \%$. A position index (PI) term is included in the $\mathrm{BI}$ to capture the effect of the position of the side chain on the reactivity. The procedure to calculate the $\mathrm{BI}$ of pure compounds, blends and those of real fuels is described in detail in our previous works $[37,69]$. A spread-sheet (named as functional group calculator) containing a number of pure compounds and standard real fuels, which can be used to calculate the input features (functional groups, molecular weight and $\mathrm{BI}$ ), is provided as Supplementary Material.

\subsection{ANN Methodology}

ANN consists of interconnected nodes similar to a group of neurons in the brain which process information by working together towards a single goal. An ANN quite simply tries to fit a function based on inputs, or "features", in order to predict an output, also referred to as "target". For this work a feed forward, densely connected, neural network was developed, trained through backpropagation. As the target is a single continuous value, the final model is considered regression-based. Overfitting is a primary problem when using ANNs. Aside from proper model validation, regularization is used to hinder this tendency. There are a few techniques available, but dropout [104] is the only method used in this work. L1 and L2 regularization, otherwise known as "lasso" and "ridge" regression, respectively, were also tested but were outperformed by dropout in early development stages and thus were removed 
from consideration. Dropout aims to prevent overfitting by randomly "dropping out" certain nodes in the network, with their corresponding weights and connections, in order to reduce both overall dependence of the network on a single node in addition to the interdependence between nodes.

ANNs can be adapted in infinitely many ways. It is important to define a realistic search space, leverage the appropriate search algorithms, in addition to having a robust validation procedure. In this work, a multi-level grid search is used to determine relatively high impact and small search space parameters, such as the number of hidden layers and the type of loss function to be employed. These parameters are discrete, and their search space has been reduced to the size where it's feasible to run grid search over all possible combinations. A genetic algorithm is leveraged to optimize the number of nodes in each layer and the respective dropout coefficients. The reasons for not incorporating these parameters into the grid search are twofold. Firstly, the search space for the number of nodes is high and the dropout coefficient is continuous - small changes can have big effects on performance. In order to reach the desired level of granularity the number of combinations required by grid search would be too computationally expensive. Secondly, the two parameters are strongly coupled. Selecting the best performing number of nodes with a fixed dropout in one level and optimizing the dropout coefficient in another led to significantly worse results on the validation sets. They need to be optimized simultaneously - a directed search leveraging a genetic algorithm achieves this without testing the large number of possible combinations.

When a dataset is large, it is realistic to simply have a dedicated validation dataset. However, the dataset in this work is relatively small, so it was desirable to maximize the size of both the training and the test sets. This was done by utilizing K-fold cross validation. The dataset was randomly split into a training and a test set. This training set was then split into $\mathrm{K}$ folds. The models were then trained on $\mathrm{K}-1$ folds and tested on the remaining. This was done until each fold has been tested a single time. The performance was based on the average of the results. The model that performed best was then finally retrained on the entire training set and then it was tested on the originally split test set.

The developed dataset consists of 499 points with 10 features and a single output, DCN. Fig. 3. illustrates the Pearson correlation between each feature and DCN. Each feature aside from "Olef$\mathrm{CH}=\mathrm{CH}_{2}{ }^{\prime \prime}$, which has a coefficient of 0.003 , have significant correlations with DCN. This preliminary observation indicates that a good model will be attainable when training on these features. Furthermore, there is no exceptionally high intra-feature correlation (redundancy) meaning that they should all increase the learning ability of the model without creating needless complexity. However, 
Paraffinic $\mathrm{CH}_{2}$ groups, molecular weight and $\mathrm{BI}$ have a slightly higher impact on the ANN model compared to the other features.

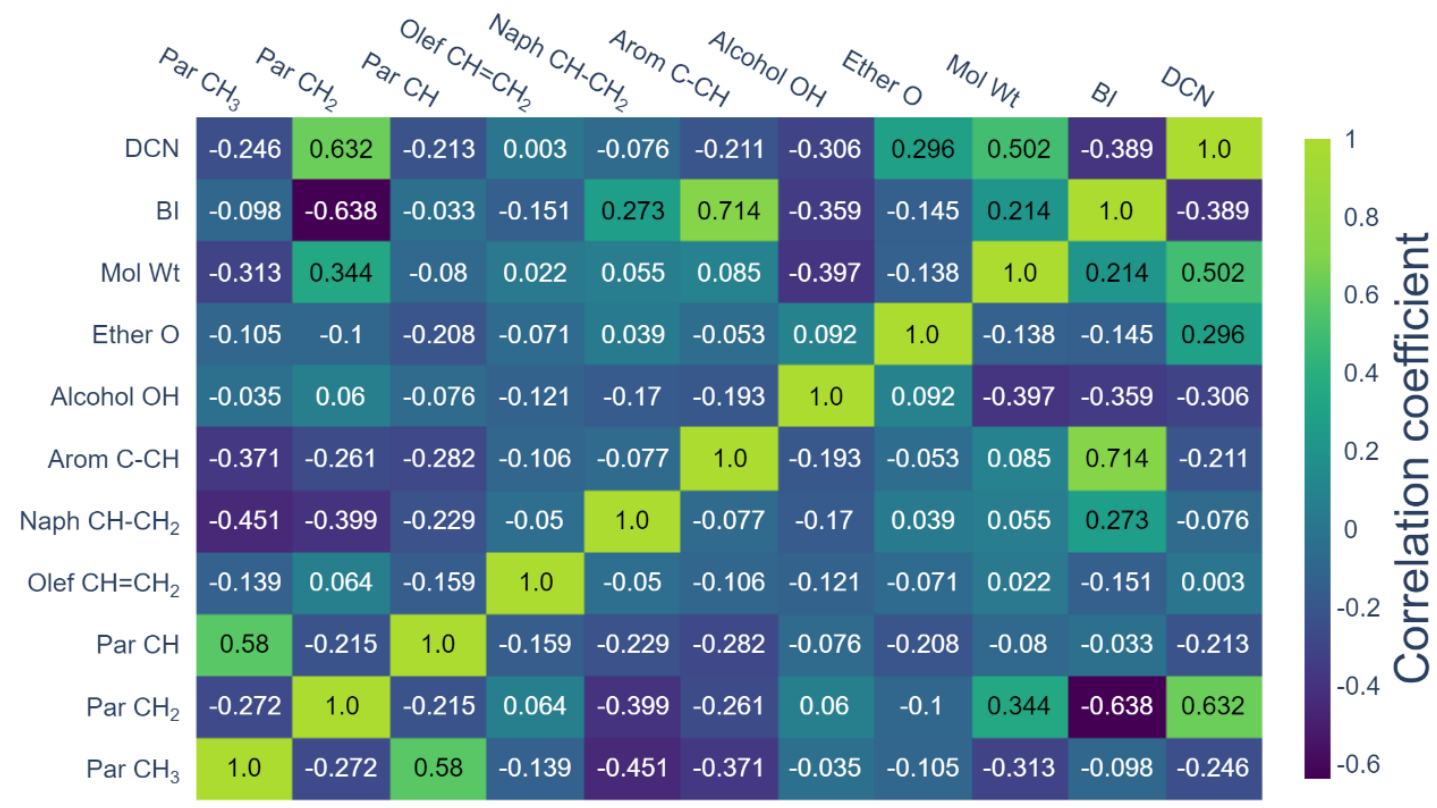

Fig. 3. Pearson correlation between the input and output features.

The training and test sets consist of 434 and 75 examples, respectively. To improve training, the top and bottom one percent of the dataset, when sorted by $\mathrm{DCN}$, were preselected to be in the training dataset. In order to facilitate learning and avoid certain features from dominating others in the loss function, the features in both sets were normalized as follows: the mean is subtracted, and the feature is scaled to unit variance. The mean, $\mu$, and variance, $\sigma$, are determined on the training set in order to avoid any information leaking from the test set. Both sets were transformed using Equation 5. Normalization was not performed on the output as there were no significant changes in model performance.

$x_{\text {scaled }}=\frac{x-\mu_{\text {train }}}{\sigma_{\text {train }}}$

The ANN was implemented using Keras [105] which is a deep learning library along with TensorFlow [106] as the backend of choice. Decisions with regards to the optimizer, initialization function, and activation function were made based on five-fold tests on the train set. Adam [107], an adaptive, gradient based optimization algorithm was the chosen optimizer because it performed well in early tests, notably better than stochastic gradient descent. The learning rate used was 0.001 . The initialization function that performed best over mean absolute percentage error was "He uniform" 
[108], and was used for all further tests. Each unit in the hidden layers receives activation, y, which is obtained by transferring the features and respective weights through summation into a rectified linear unit (ReLU) [109].

\subsubsection{Grid search and genetic algorithm}

A grid search (see Fig. S1.) was utilized to settle the number of hidden layers, batch size, loss function and number of epochs. After optimizing the hyperparameters a genetic algorithm was then employed to determine the dropout coefficients and the number of nodes. In order to reduce computational costs, the grid search was divided into four levels. The complete search space is presented in Table 5. The levels were sorted in descending order sorted by perceived relative importance to the performance of the model. The number of hidden layers was targeted first. Meaning that the loss functions, epoch number, and batch size were all kept as variables, but with a search spaces as strict subsets of their entire spaces - for example batch sizes varied between $\{4,64$, and 512$\}$. By allowing these parameters to vary a little bit the chance that, in this case, the number of hidden layers is chosen based on the values of the other parameters is reduced. Moving forward, the hidden layer number was fixed, and the search was focused on loss functions, proceeding by grid search, until all parameters were fixed and only the number of epochs is being searched over. As before, five-fold cross validation was used as the evaluation metric for the combination of various hyperparameters. The combination that resulted in the lowest mean average absolute error was used then chosen to pick the value of each hyperparameter.

\section{Table 5}

Hyperparameter Space

\begin{tabular}{ll}
\hline Parameter & Possible Values \\
\hline Number of hidden layers & $\{x \in N \mid 1=<x<=4\}$ \\
Number of units per layer & $\{x \in N \mid 10=<x<=640\}$ \\
Number of epochs & $\{500 x \in N \mid 1=<x<=10\}$ \\
Batch size & $\{4,8,16,32,64,128,256,512\}$ \\
Loss function & RMSE; Mean absolute error, Mean absolute percentage error, \\
& Mean squared logarithmic error. \\
Dropout rate & $\{x \in R \mid 0=<x<=0.8\}$ \\
\hline
\end{tabular}

Once the above hyperparameters were determined, a genetic algorithm was applied for the determination of number of units per hidden layer in addition to the respective dropout coefficients. To this end, a single ANN is treated as an individual. Four of the parameters contained in this individual are 
of interest to the genetic algorithm: the number of units and dropout coefficients for the two hidden layers. Further details on the genetic algorithm are provided in the Supplementary Material.

\section{RESULTS \& DISCUSSION}

The following sections discuss the effect of alcohol $\mathrm{OH}$ and ether $\mathrm{O}$ groups in the fuel on its ignition quality. The effect of the other six functional groups on DCN has been discussed in detail in our previous work [37]. Paraffinic $\mathrm{CH}_{3}$ groups in the fuel have a negative impact on it DCN. Experimental ignition delay measurements of various mixtures of $C_{16}$ iso-paraffin mixtures showed a decrease in the DCN when the methyl content (paraffinic $\mathrm{CH}_{3}$ groups) of the mixtures was increased [67]. A similar effect was also observed in blends consisting of n-heptane and 2,2,4-trimethylpentane, 1-hexene and cyclohexane. Mass fractions of paraffinic $\mathrm{CH}_{2}$ groups have a significant impact on the combustion behavior of paraffinic fuels [76]. There is a linear increase in the DCN of a fuel when the wt \% of paraffinic $\mathrm{CH}_{2}$ groups increases, as observed in a number of pure compounds and blends of $n$-heptane, 2,2,4-trimethylpentane and toluene. The ratio of paraffinic $\mathrm{CH}_{2} / \mathrm{CH}_{3}$ groups is an important criterion in the auto-ignition of paraffinic fuels [67] and has a good correlation with ignition delay [41]. Paraffinic $\mathrm{CH}$ groups generally have a positive effect on IDT and negative effect on DCN, as they possess low bond dissociation energies. Paraffinic $\mathrm{CH}$ groups, which are a result of the presence of side chains, increase the octane rating and reduce the cetane rating. Olefins have a lower DCN value compared to n-paraffins of similar carbon numbers as olefinic $-\mathrm{CH}=\mathrm{CH}_{2}$ groups are less reactive compared to their saturated counterparts. Naphthenes, which have cyclic structures, have similar molecular formulas compared to olefins but exhibit significant differences in their combustion chemistry which are similar to n-paraffins. In general, naphthenes exhibit lower DCN compared to paraffins and olefins possessing similar carbon numbers [37]. Aromatic $\mathrm{C}-\mathrm{CH}$ groups have a marked effect on $\mathrm{DCN}$ and diminish the $\mathrm{DCN}$ more severely than other groups as the aromatic rings form stable intermediate radicals that increase the IDT of the oxidation reactions [66]. The addition of a linear paraffinic chain to an aromatic ring increases the DCN of the molecule [37].

\subsection{Alcohol $\mathrm{OH}$ groups}

Alcohol $\mathrm{OH}$ groups are known to lower fuel reactivity as they promote the formation of radical scavenging intermediates that reduce LTHR [24]. Therefore, an increase in alcohol OH content of a fuel results in the decrease of its DCN. It has been experimentally shown that ethanol's $\mathrm{OH}$ groups have a greater effect than aromatic groups on the octane number of a fuel [66]. A unit increase (wt \%) in 
ethanol content results in a greater increase in the motor octane number (MON) and the research octane number (RON) than any other hydrocarbon functional group because the $\mathrm{OH}$ group adjacent to the $\mathrm{CH}_{2}$ group weakens its bond strength, favoring $\mathrm{H}$-abstraction. The effect of alcohol $\mathrm{OH}$ group on a number of pure compounds is shown in Fig. 4. and it can be observed that an increase in the OH group wt\% results in a decrease in the DCN. Alcohol compounds generally have low DCNs compared to paraffins or olefins of the same carbon number. For example, 1-heptanol has a lower DCN (29) compared to n-heptane (DCN, 53.8) and 1-heptene (DCN, 32). Methanol has the lowest DCN of 6.1 of any alcohol compound in the dataset. It also has the highest $\mathrm{OH}$ content of $53.1 \mathrm{wt} \%$.

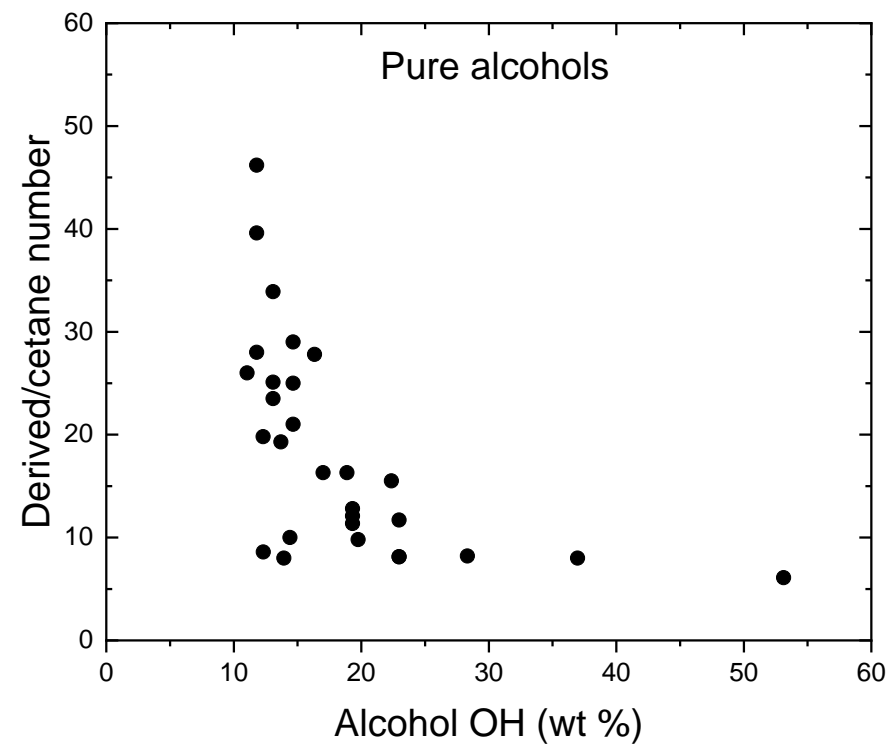

Fig. 4. Effect of alcohol OH groups on DCN of pure compounds

The effects of alcohol OH groups on DCN of blends containing various PRFs with pure alcohols are shown in Figs. 5, 6 and 7. Fig. 8. shows the effect of alcohol OH groups on DCN of blends containing various TPRFs with pure alcohols and Fig. 9. shows the effect of alcohol OH groups on blends of DEE with various pure alcohols. It can be seen throughout this figure that as the alcohol $\mathrm{OH}$ content is gradually increased in the blends the DCN continues to fall steadily. DEE has a DCN of 139 and when $10 \%$ (by volume) of methanol is added to DEE the mixture DCN becomes 97.2. Similarly when $10 \%$ ethanol and $n$-butanol are blended with DEE, the measured DCN values are 97.7 and 101.9 respectively. On the first look, Fig. 9. shows a more rapid DCN reduction for $\mathrm{n}$-butanol and ethanol addition compared to methanol because the horizontal abscissa of the figure is expressed in $\mathrm{OH}$ wt \% and methanol has a higher $\mathrm{OH}$ wt $\%$ per molecule compared to both ethanol and $\mathrm{n}$-butanol. 


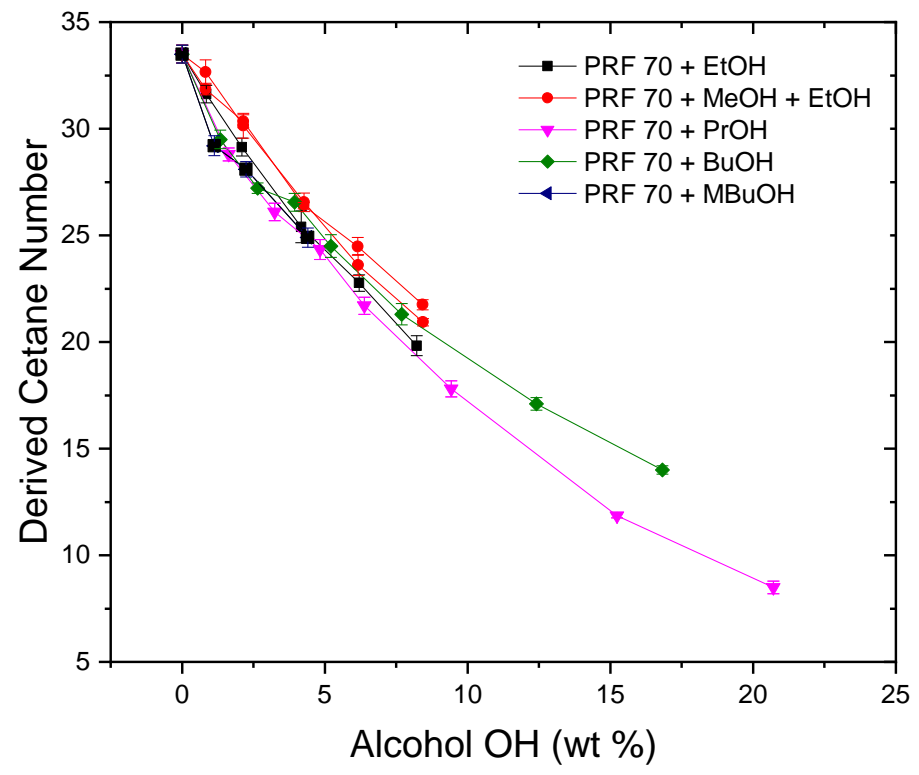

Fig. 5. Effect of alcohol OH groups on DCN of blends containing PRF 70 with various alcohols.

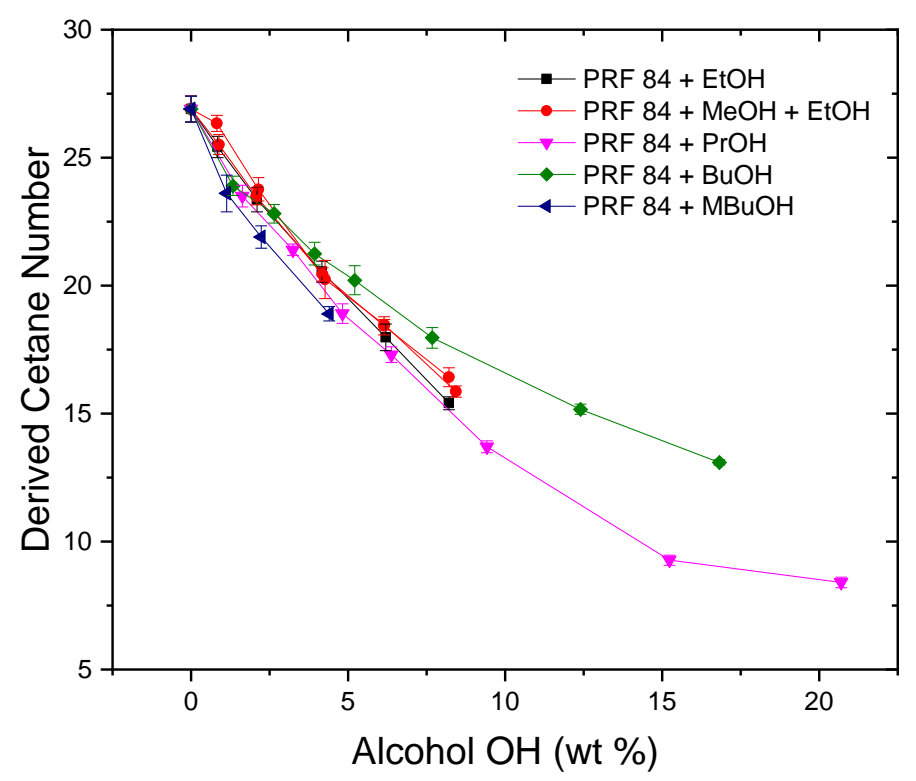

Fig. 6. Effect of alcohol OH groups on DCN of blends containing PRF 84 with various alcohols. 


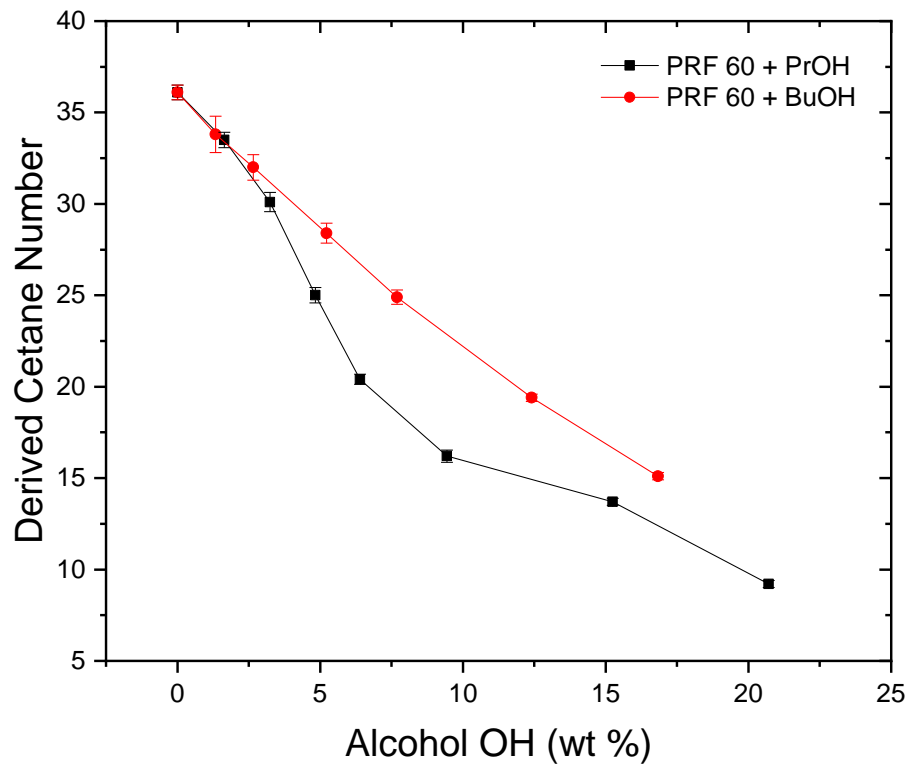

Fig. 7. Effect of alcohol OH groups on DCN of blends containing PRF 60 with various alcohols.

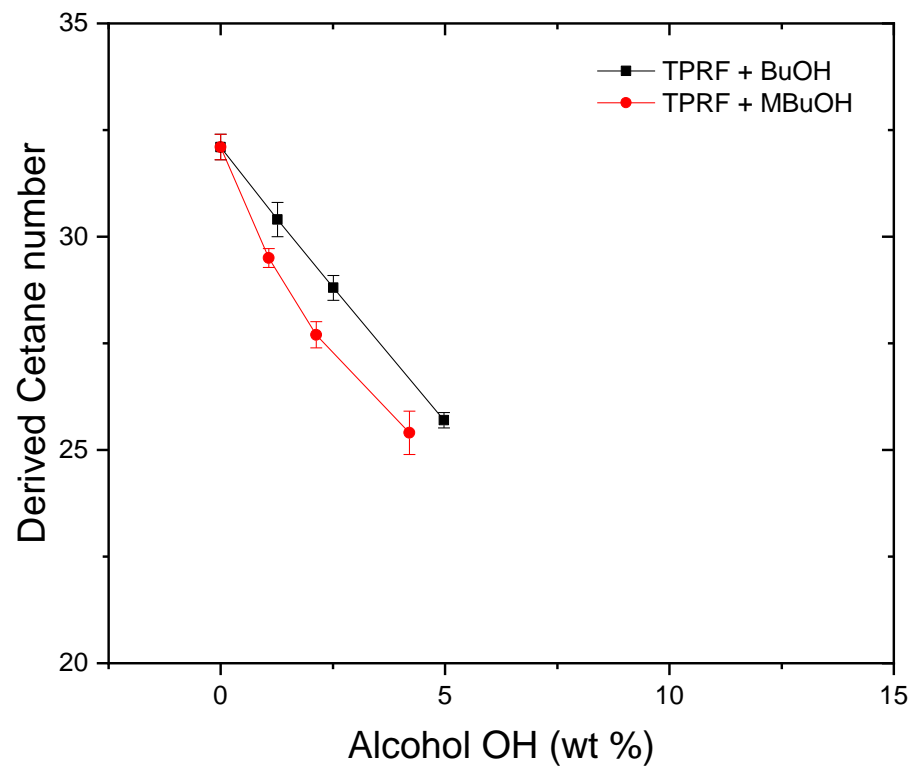

Fig. 8. Effect of alcohol OH groups on DCN of blends containing TPRF with various alcohols. 


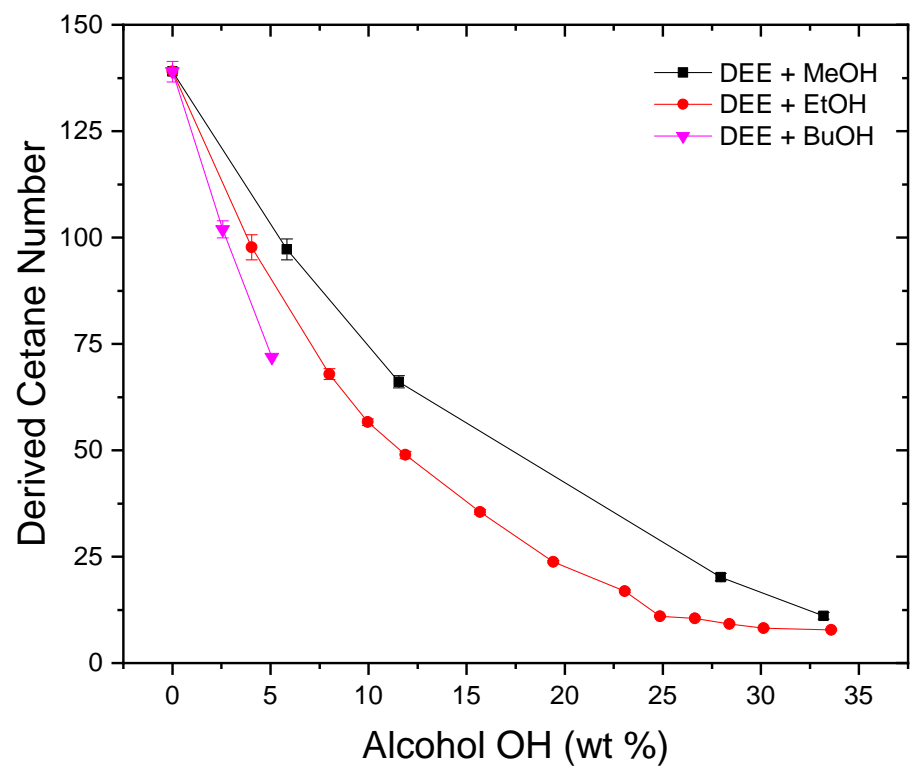

Fig. 9. Effect of alcohol $\mathrm{OH}$ groups on $\mathrm{DCN}$ of blends containing DEE with various alcohols.

DCN of a number of real fuels (FACE gasoline's A, I and J) blended with various pure alcohols was experimentally measured in the IQT and the change in DCN with corresponding change in the alcohol $\mathrm{OH}$ content is shown in Figs. 10, 11 and 12. As expected, the DCN reduces with increasing alcohol $\mathrm{OH}$ content.

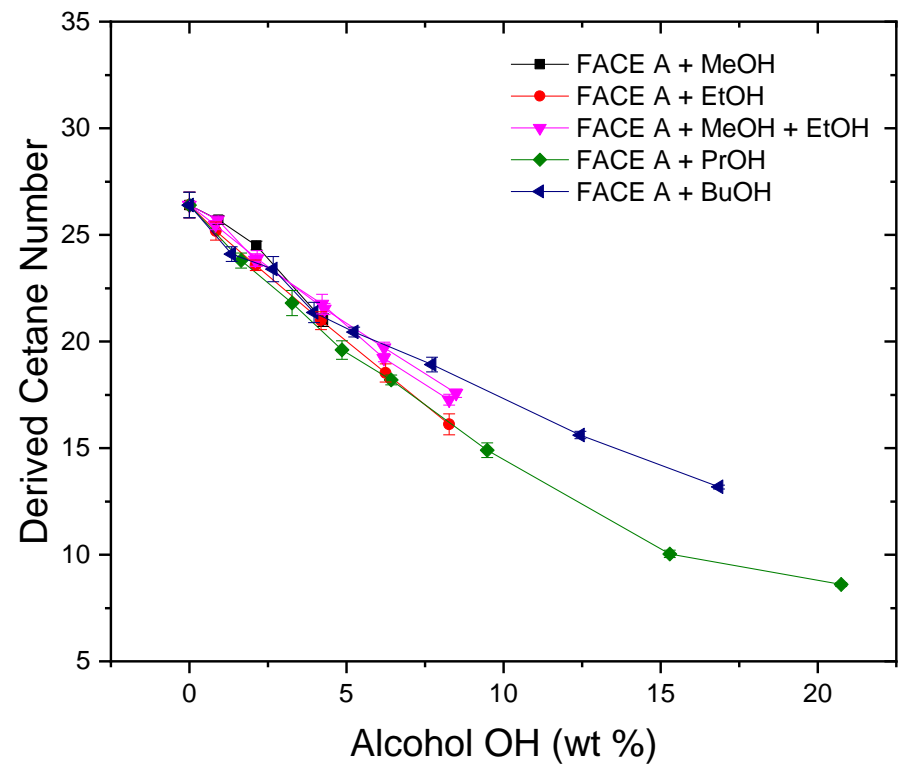

Fig. 10. Effect of alcohol OH groups on DCN of blends containing FACE A gasoline with various alcohols. 


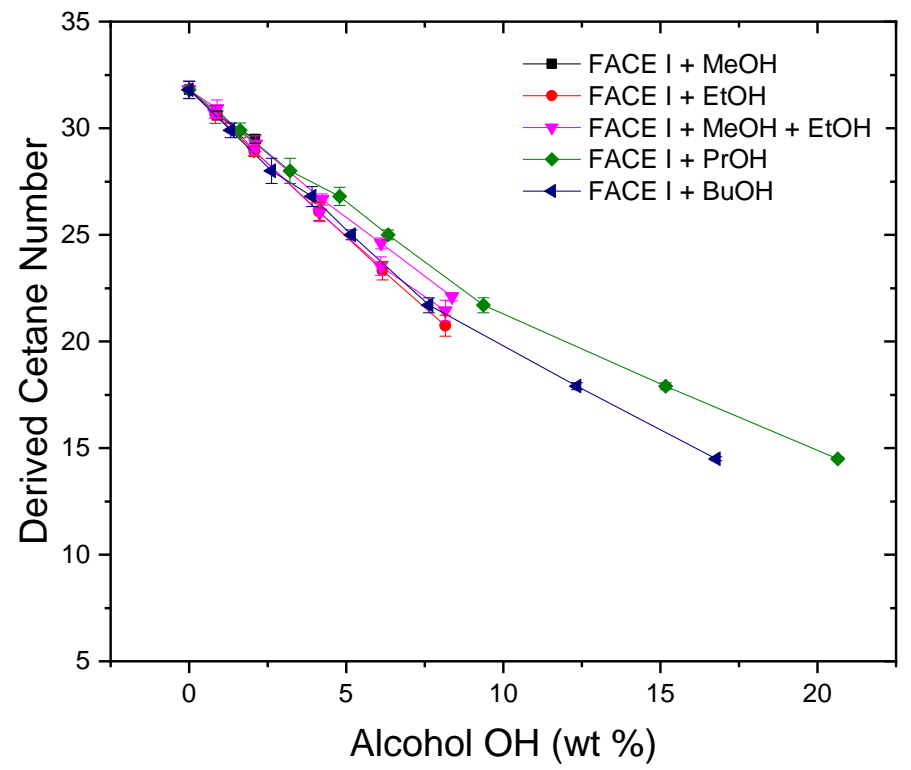

Fig. 11. Effect of alcohol OH groups on DCN of blends containing FACE I gasoline with various alcohols.

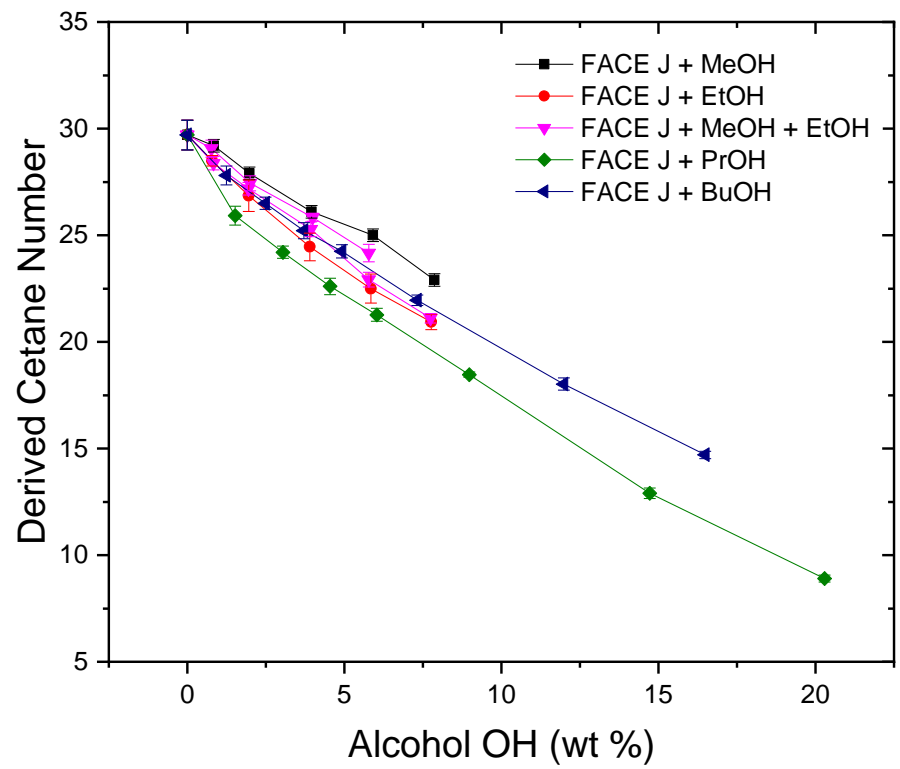

Fig. 12. Effect of alcohol OH groups on DCN of blends containing FACE J gasoline with various alcohols.

\subsection{Ether $O$ groups}

The oxidation chemistry of ethers is treated with rules close to that of paraffins as shown by a number of studies made on the oxidation of methyl-tert-butyl-ether [110], ethyl-tert-butyl-ether [111] , tertamyl-methyl-ether [111] and dimethylether [112]. The molecular $\mathrm{H}$ transfer reaction from the carbon atom in the beta position to the ether $\mathrm{O}$ atom to yield an alkyl radical and alcohol molecule seems to be the only reaction exclusive to the ethers [113] and it is very sensitive to wall effects. Ether compounds in 
a fuel are also good at reducing particulate matter emissions and an investigation by Liotta and Montalvo [114] showed that $O$ atoms in ether functional groups were more efficient at reducing soot compared to $\mathrm{O}$ atoms in alcohol functional groups. Fig. 13. shows the effect of ether $\mathrm{O}$ groups on the DCN of pure compounds. The DCN of ether compounds depends not only the contribution made by the ether functional groups but also on other constituent functional groups, molecular weight and $\mathrm{BI}$ of the molecule. DEE has the highest DCN of 139 [115] in the dataset followed by DBE which has a DCN of 115.2 [116] and these have been used as cetane improvers. The ether data points used in other DCN models [33] reported in the literature are usually made up of pure compounds and the number of such data available is limited and close to 20 . The present fuel dataset contains 92 datapoints representing neat ethers or fuel blends containing ethers. These can effectively capture the effect of the ether functional group on the resulting DCN, which can be applied to new fuels containing ethers. This is a limitation observed for other ANN-based, DCN prediction models [33].

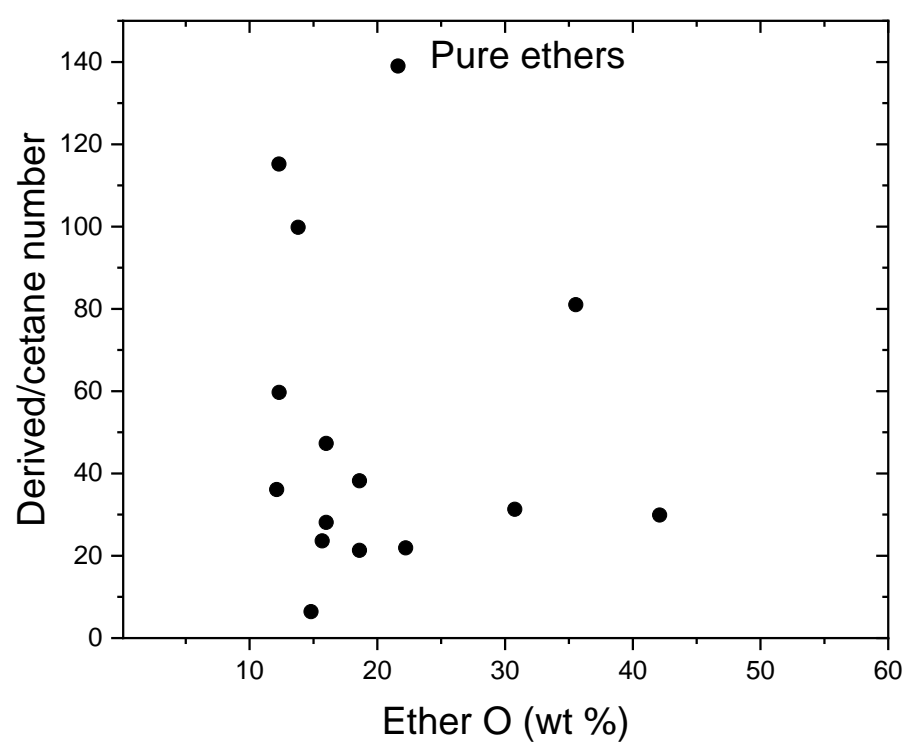

Fig. 13. Effect of ether O groups on DCN of pure compounds

The effect of ether $\mathrm{O}$ groups on DCN of blends of DEE with a number of pure compounds is shown in Fig. 14. and it can be seen that the DCN increases with increasing ether O groups. The DCN of $n$-heptane and iso-octane increases steadily when DEE is blended with them. The DCN of alcohol compounds namely, methanol and ethanol exhibited a very gradual increase at first and then a steady rise was observed when DEE volumetric percentage was increased beyond $50 \%$. 


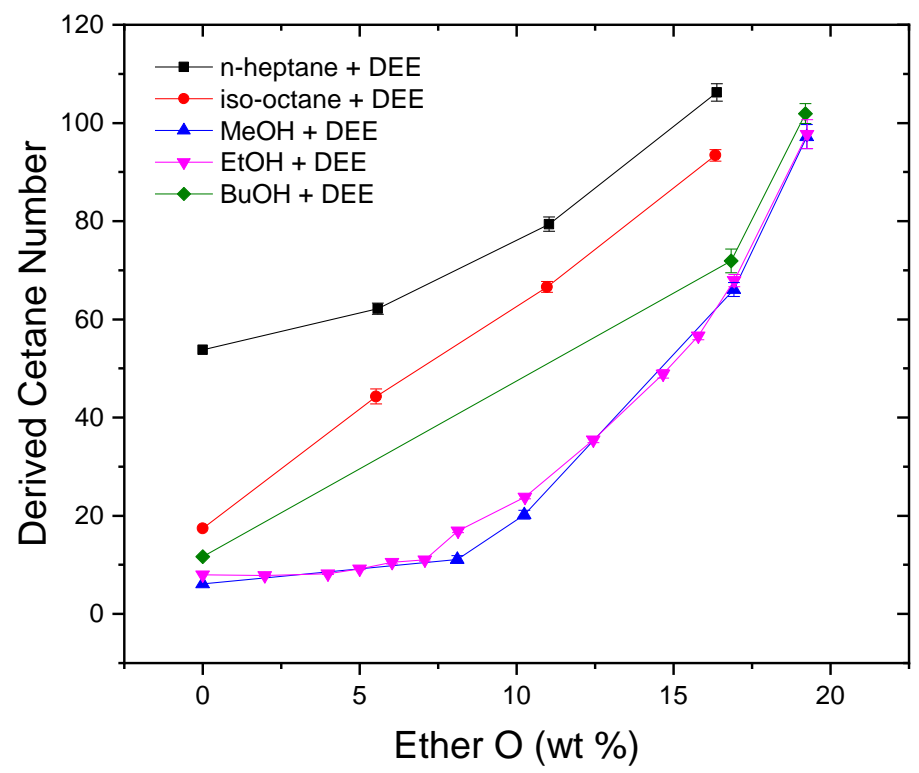

Fig. 14. Effect of ether O groups on DCN of blends containing DEE with various compounds

Figs. 15, 16 and 17 show the variation of DCN of a number of real fuels namely SG (DCN, 19.2), LN J80 (DCN, 27.6) and HN J80 (DCN, 23.6) respectively, when blended with various ether compounds like DEE, DPE, DIPE, DBE and DMM. The DCN values of the real fuels were found to increase due to the increase in the ether $\mathrm{O}$ groups in all the cases except for the fuels blended with DIPE. A 1\% (by volume) addition of DIPE to SG, LN J80 and HN J80 led to an increase of the DCN of the blends to 19.5, 29.3 and 32.3, respectively. Further increase in the volumetric blending of DIPE to 5, 10 and $20 \%$ led to a decrease in the DCN of all the three fuels. This shows a synergistic blending effect on DCN when DIPE was added to the fuels in minute quantities (1\%) whereas an antagonistic effect was observed when the proportion of DIPE was increased beyond $1 \%$. 


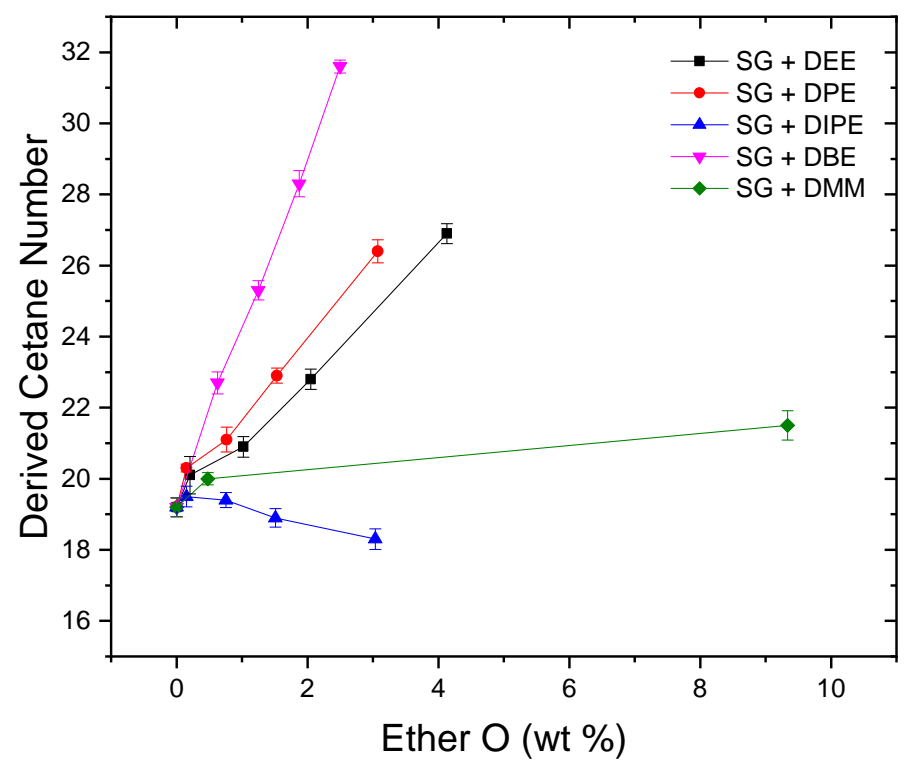

Fig. 15. Effect of ether O groups on DCN of blends containing SG with various ethers

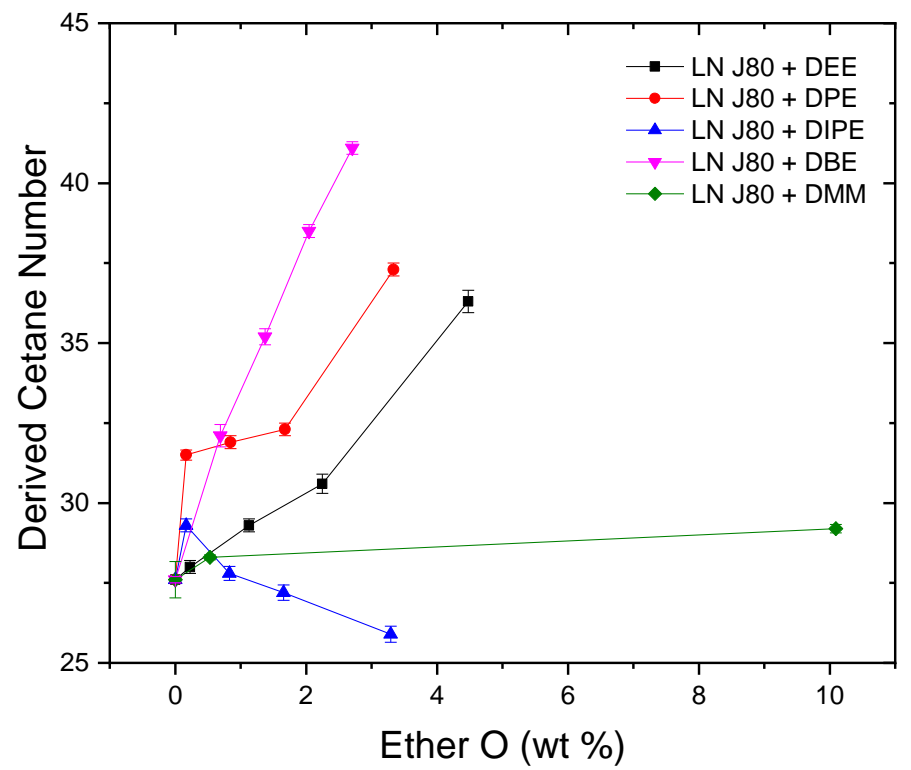

Fig. 16. Effect of ether $\mathrm{O}$ groups on $\mathrm{DCN}$ of blends containing $L N J 80$ with various ethers 


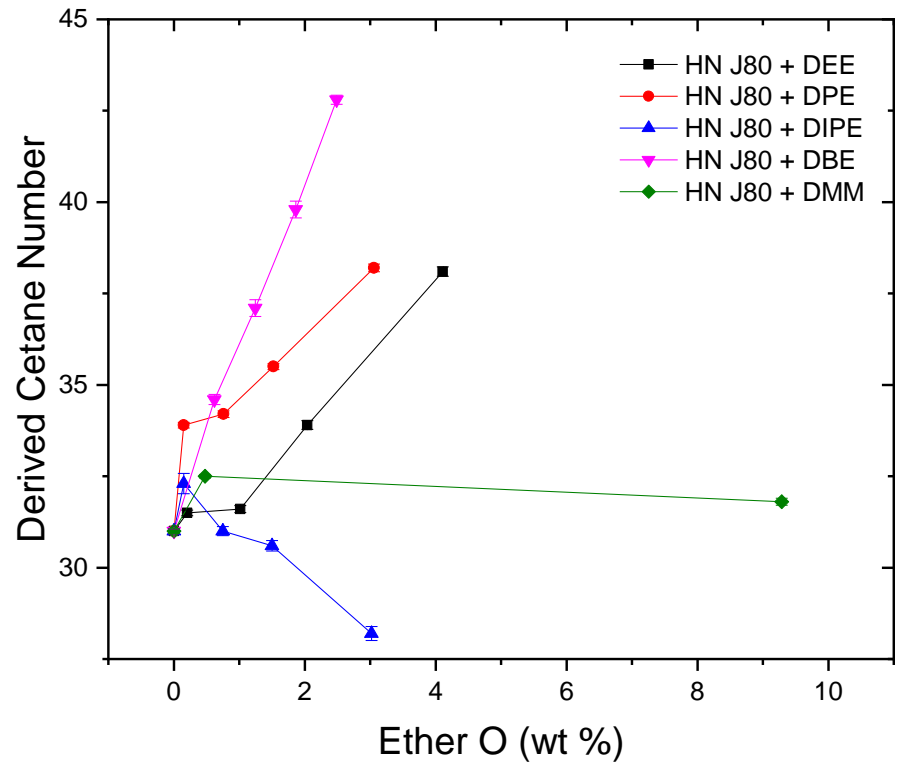

Fig. 17. Effect of ether $\mathrm{O}$ groups on $\mathrm{DCN}$ of blends containing $\mathrm{HN} \mathrm{J80}$ with various ethers

\subsection{ANN Model}

An ANN model was developed, following the described methodology in section 3.2, to predict DCN as a function of the input features. Hyperparameters in the model were determined using a grid search method, followed by a genetic algorithm to optimize the ANN structure. The final hyperparameters of the model, i.e., number of hidden layers, loss function, batch size, epochs, number of nodes per layer, and the dropout coefficients, are shown in Table 6. The final architecture of the model is represented, in part, in Fig. 18.

Input layer Hidden layer $1 \quad$ Hidden layer $2 \quad$ Output layer

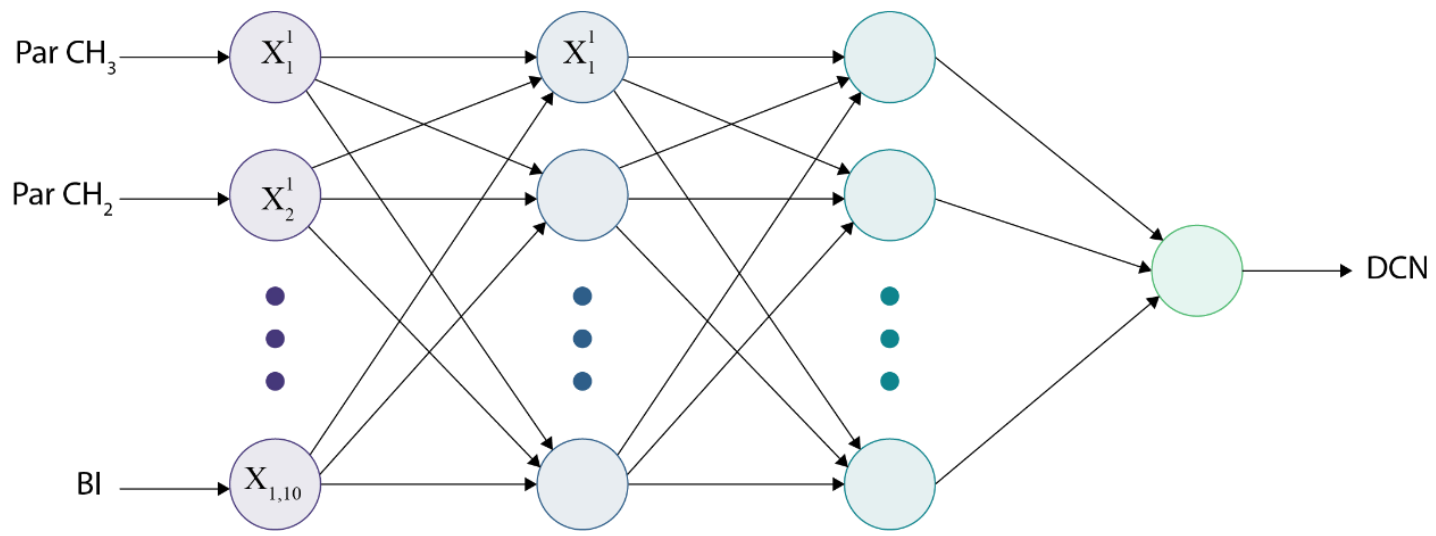

Fig. 18. Graphical representation of the final ANN's architecture 
The final model is the average prediction of ten individual models in order to mitigate any results arising from random initialization, allowing for more stable results. Essentially, each ANN model was trained ten times on the entire training set from randomly initialized weights, and an average of the predictions on the test set was used to determine the final model's performance. Note that this test set is only used for final predictions and was never used in order to tune the model in any way. The scores for the various error metrics of interest are presented in Table 7. The average absolute error is near the limit of uncertainty for DCN measurements and the average percentage error is also small. Fig. 19. shows the predictions on the test set vs. the expected values plotted against each other, with a straight line representing the ideal case. Each result in Fig. 19. is colored by the log percentage error. The complete dataset, training set and the test set with the predictions are provided as Supplementary Material.

\section{Table 6}

Hyperparameters of the final model

\begin{tabular}{lll}
\hline Parameter & Hidden layer 1 & Hidden layer 2 \\
\hline Dropout coefficient & 0.135 & 0.041 \\
Number of units & 442 & 290 \\
\cline { 2 - 3 } Number of hidden layers & 2 \\
Batch size & 32 \\
Loss function & Mean absolute percentage error \\
Number of epochs & 3000 \\
\hline
\end{tabular}

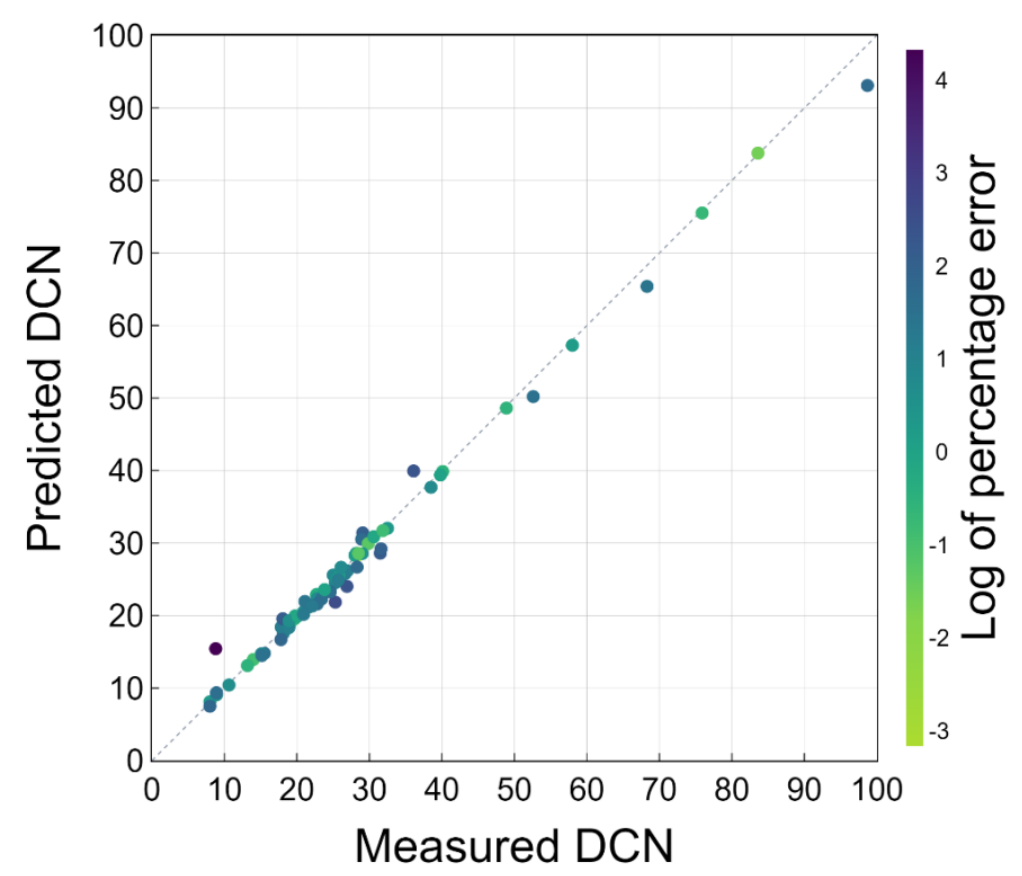

Fig. 19. Comparison between the predicted and the measured DCN values in the test set which include pure components, blends of pure components and real fuel blend 
Table 7

Summary of results on the test set

\begin{tabular}{ll}
\hline Error metric & Result \\
\hline Average absolute error & 0.91 \\
Average percentage error & $4.00 \%$ \\
RMSE & 1.51 \\
$\mathrm{R}^{2}$ & 0.992 \\
\hline
\end{tabular}

The final metrics for the performance of the model on the test set are shown in Table 7. The developed ANN model for DCN prediction has a low average absolute error of 0.91 compared to other DCN models with absolute errors of 1.25 [54], 1.47 [53], 2 [57], 2.11 [51], 5.8 [39], 6.9 [35], 7.1 [34] etc. The average absolute error obtained depends on the training and test sets used, modeling approaches and the variety of chemical classes covered and therefore the present model does not claim superiority over other DCN models in lieu of the low error. Some $[35,51,53,54]$ of these DCN models are valid only for non-oxygenated fuels whose fuel chemistry is less complex whilst other models $[34,39]$ cover a wider range of oxygenates including esters, ketones, acids and aldehydes. The present model can predict DCN of pure compounds, pure hydrocarbon and oxygenate blends, and oxygenated petroleum fuels containing alcohol and ether functionalities as these oxygenated chemical classes are more commonly used in diesel fuels as additives. The final model is available on the GitHub repository: https://github.com/vcovo/dcn-prediction

\section{CONCLUSIONS}

ANN modeling was applied to predict the derived cetane number (DCN) of oxygenated fuels containing oxygenate alcohol and ether functionalities. Experimental DCNs of 499 fuels comprising of 116 pure compounds, 222 pure compound blends and 159 real fuel blends were used in the dataset for training and testing the model. Sixty new DCN measurements were carried out in this work. Fuel chemical composition expressed in the form of eight functional groups namely paraffinic $\mathrm{CH}_{3}$ groups, paraffinic $\mathrm{CH}_{2}$ groups, paraffinic $\mathrm{CH}$ groups, olefinic $-\mathrm{CH}=\mathrm{CH}_{2}$ groups, naphthenic $\mathrm{CH}-\mathrm{CH}_{2}$ groups, aromatic $\mathrm{C}-\mathrm{CH}$ groups, alcoholic $\mathrm{OH}$ groups and ether $\mathrm{O}$ groups, along with two structural parameters namely, molecular weight and branching index (BI), were used as the ten input features of the model. Functional groups of real fuels were qualitatively and quantitatively analyzed using ${ }^{1} \mathrm{H}$ nuclear magnetic resonance (NMR) spectroscopy. A multi-level grid search was used to determine optimum hyperparameters such as the number of hidden layers, type of loss function, batch size and total number of epochs to be 
employed. A genetic algorithm was then applied to optimize the number and the respective dropout coefficients. Three input features namely the paraffinic $\mathrm{CH}_{3}$ groups, molecular weight and $\mathrm{BI}$ have a slightly more significant impact on the ANN model compared to the functional groups as shown by the Pearson correlation heatmap. The final ANN model developed was tested with $15 \%$ of unseen randomly generated data points and a regression coefficient $\left(R^{2}\right)$ of 0.992 was obtained for the test set along with an average absolute error of 0.91 . The errors associated with blends of real fuels and ether compounds, and those with multicomponent blends with more than three components were more than 2-3 times the average error. This is because of the high diversity of functional groups in these blends and the uncertainty associated with functional group quantification of real from ${ }^{1} \mathrm{H} N M R$. Overall the developed ANN model is successful in predicting the DCN of oxygenated fuels (alcohols and ethers classes only) and can be used to predict the DCN of pure compounds, pure hydrocarbon and oxygenate blends, and oxygenated petroleum transportation fuels.

\section{Acknowledgement}

\section{References}

1. Reitz, R.D., Ogawa, H., Payri, R., Fansler, T., Kokjohn, S., Moriyoshi, Y., Agarwal, A., Arcoumanis, D., Assanis, D., Bae, C., Boulouchos, K., Canakci, M., Curran, S., Denbratt, I., Gavaises, M., Guenthner, M., Hasse, C., Huang, Z., Ishiyama, T., Johansson, B., Johnson, T., Kalghatgi, G., Koike, M., Kong, S., Leipertz, A., Miles, P., Novella, R., Onorati, A., Richter, M., et al., "IJER editorial: The future of the internal combustion engine," Int. J. Engine Res. 21(1):3-10, 2020, doi:10.1177/1468087419877990.

2. Kalghatgi, G., "Is it really the end of internal combustion engines and petroleum in transport?," Appl. Energy 225(February):965-974, 2018, doi:10.1016/j.apenergy.2018.05.076.

3. Kalghatgi, G., "Development of Fuel/Engine Systems-The Way Forward to Sustainable Transport," Engineering 5(3):510-518, 2019, doi:10.1016/j.eng.2019.01.009.

4. Singh, E., Badra, J., Mehl, M., and Sarathy, S.M., "Chemical Kinetic Insights into the Octane Number and Octane Sensitivity of Gasoline Surrogate Mixtures," Energy \& Fuels 31(2):19451960, 2017, doi:10.1021/acs.energyfuels.6b02659.

5. ASTM D613 Standard Test Method for Cetane Number of Diesel Fuel Oil, 2015, doi:10.1520/D0613.

6. ASTM D6890 Standard Test Method for Determination of Ignition Delay and Derived Cetane Number ( DCN ) of Diesel Fuel Oils by Combustion in a Constant Volume Chamber, 2013.

7. Perez, P.L. and Boehman, A.L., "Experimental Investigation of the Autoignition Behavior of Surrogate Gasoline Fuels in a Constant-Volume Combustion Bomb Apparatus and Its Relevance to HCCl Combustion," Energy \& Fuels 26(10):6106-6117, 2012, doi:10.1021/ef300503b.

8. Yanowitz, J., Ratcliff, M.A., McCormick, R.L., Taylor, J.D., and Murphy, M.J., “Compendium of 
Experimental Cetane Numbers," Golden, CO (United States), 2017, doi:10.2172/1345058.

9. Naser, N., Sarathy, S.M., and Chung, S.H., "Ignition delay time sensitivity in ignition quality tester (IQT) and its relation to octane sensitivity," Fuel 233(January):412-419, 2018, doi:10.1016/j.fuel.2018.05.131.

10. Naser, N., Yang, S.Y., Kalghatgi, G., and Chung, S.H., "Relating the octane numbers of fuels to ignition delay times measured in an ignition quality tester (IQT)," Fuel 187:117-127, 2017, doi:10.1016/j.fuel.2016.09.013.

11. Won, S.H., Rock, N., Lim, S.J., Nates, S., Carpenter, D., Emerson, B., Lieuwen, T., Edwards, T., and Dryer, F.L., "Preferential vaporization impacts on lean blow-out of liquid fueled combustors," Combust. Flame 205:295-304, 2019, doi:10.1016/j.combustflame.2019.04.008.

12. Liu, H., Wang, Z., Li, Y., Zheng, Y., He, T., and Wang, J., "Recent progress in the application in compression ignition engines and the synthesis technologies of polyoxymethylene dimethyl ethers," Appl. Energy 233-234(June 2018):599-611, 2019, doi:10.1016/j.apenergy.2018.10.064.

13. Li, B., Li, Y., Liu, H., Liu, F., Wang, Z., and Wang, J., "Combustion and emission characteristics of diesel engine fueled with biodiesel/PODE blends," Appl. Energy 206:425-431, 2017, doi:10.1016/j.apenergy.2017.08.206.

14. Liu, H., Wang, Z., Zhang, J., Wang, J., and Shuai, S., "Study on combustion and emission characteristics of Polyoxymethylene Dimethyl Ethers/diesel blends in light-duty and heavy-duty diesel engines," Appl. Energy 185:1393-1402, 2017, doi:10.1016/j.apenergy.2015.10.183.

15. Liu, J., Sun, P., Huang, H., Meng, J., and Yao, X., "Experimental investigation on performance, combustion and emission characteristics of a common-rail diesel engine fueled with polyoxymethylene dimethyl ethers-diesel blends," Appl. Energy 202:527-536, 2017, doi:10.1016/j.apenergy.2017.05.166.

16. Kessler, T., Sacia, E.R., Bell, A.T., and Mack, J.H., "Artificial neural network based predictions of cetane number for furanic biofuel additives," Fuel 206:171-179, 2017, doi:10.1016/j.fuel.2017.06.015.

17. Herreros, J.M., Jones, A., Sukjit, E., and Tsolakis, A., "Blending lignin-derived oxygenate in enhanced multi-component diesel fuel for improved emissions," Appl. Energy 116:58-65, 2014, doi:10.1016/j.apenergy.2013.11.022.

18. Vallinayagam, R., Vedharaj, S., Yang, W.M., Raghavan, V., Saravanan, C.G., Lee, P.S., Chua, K.J.E., and Chou, S.K., "Investigation of evaporation and engine characteristics of pine oil biofuel fumigated in the inlet manifold of a diesel engine," Appl. Energy 115:514-524, 2014, doi:https://doi.org/10.1016/j.apenergy.2013.11.004.

19. PEPIOTDESJARDINS, P., PITSCH, H., MALHOTRA, R., KIRBY, S., and BOEHMAN, A., "Structural group analysis for soot reduction tendency of oxygenated fuels," Combust. Flame 154(1-2):191205, 2008, doi:10.1016/j.combustflame.2008.03.017.

20. Gao, Z., Zou, X., Huang, Z., and Zhu, L., "Predicting sooting tendencies of oxygenated hydrocarbon fuels with machine learning algorithms," Fuel 242:438-446, 2019, doi:10.1016/J.FUEL.2019.01.064.

21. Das, D.D., John, P.C. St., McEnally, C.S., Kim, S., and Pfefferle, L.D., “Measuring and predicting 
sooting tendencies of oxygenates, alkanes, alkenes, cycloalkanes, and aromatics on a unified scale," Combust. Flame 190:349-364, 2018, doi:10.1016/J.COMBUSTFLAME.2017.12.005.

22. Barrientos, E.J., Lapuerta, M., and Boehman, A.L., "Group additivity in soot formation for the example of C-5 oxygenated hydrocarbon fuels," Combust. Flame 160(8):1484-1498, 2013, doi:10.1016/j.combustflame.2013.02.024.

23. Abdul Jameel, A.G., Alkhateeb, A., Telalović, S., Elbaz, A.M., Roberts, W.L., and Sarathy, S.M., "Environmental Challenges and Opportunities in Marine Engine Heavy Fuel Oil Combustion," Proceedings of the Fourth International Conference in Ocean Engineering, Springer, ISBN 9789811331190: 1047-1055, 2019, doi:10.1007/978-981-13-3119-0_72.

24. Sarathy, S.M., Oßwald, P., Hansen, N., and Kohse-Höinghaus, K., "Alcohol combustion chemistry," Prog. Energy Combust. Sci. 44:40-102, 2014, doi:10.1016/j.pecs.2014.04.003.

25. Labeckas, G., Slavinskas, S., and Kanapkienè, I., "The individual effects of cetane number, oxygen content or fuel properties on performance efficiency, exhaust smoke and emissions of a turbocharged CRDI diesel engine - Part 2," Energy Convers. Manag. 149:442-466, 2017, doi:https://doi.org/10.1016/j.enconman.2017.07.017.

26. Labeckas, G., Slavinskas, S., and Kanapkienè, I., "The individual effects of cetane number, oxygen content or fuel properties on the ignition delay, combustion characteristics, and cyclic variation of a turbocharged CRDI diesel engine - Part 1," Energy Convers. Manag. 148:1003-1027, 2017, doi:https://doi.org/10.1016/j.enconman.2017.06.050.

27. Zhou, X., Qian, W., Pan, M., Huang, R., Xu, L., and Yin, J., "Potential of n-butanol/diesel blends for $\mathrm{Cl}$ engines under post injection strategy and different EGR rates conditions," Energy Convers. Manag. 204:112329, 2020, doi:https://doi.org/10.1016/j.enconman.2019.112329.

28. Santhosh, K. and Kumar, G.N., "Impact of 1-Hexanol/diesel blends on combustion, performance and emission characteristics of CRDI Cl mini truck engine under the influence of EGR," Energy Convers. Manag. 217:113003, 2020, doi:https://doi.org/10.1016/j.enconman.2020.113003.

29. Nanthagopal, K., Kishna, R.S., Atabani, A.E., Al-Muhtaseb, A.H., Kumar, G., and Ashok, B., “A compressive review on the effects of alcohols and nanoparticles as an oxygenated enhancer in compression ignition engine," Energy Convers. Manag. 203:112244, 2020, doi:https://doi.org/10.1016/j.enconman.2019.112244.

30. Battin-Leclerc, F., "Detailed chemical kinetic models for the low-temperature combustion of hydrocarbons with application to gasoline and diesel fuel surrogates," Prog. Energy Combust. Sci. 34(4):440-498, 2008, doi:10.1016/j.pecs.2007.10.002.

31. Mohebbi, M., Reyhanian, M., Hosseini, V., Said, M.F.M., and Aziz, A.A., "The effect of diethyl ether addition on performance and emission of a reactivity controlled compression ignition engine fueled with ethanol and diesel," Energy Convers. Manag. 174:779-792, 2018, doi:https://doi.org/10.1016/j.enconman.2018.08.091.

32. Channapattana, S.V., Pawar, A.A., and Kamble, P.G., "Optimisation of operating parameters of DI$\mathrm{Cl}$ engine fueled with second generation Bio-fuel and development of ANN based prediction model," Appl. Energy 187:84-95, 2017, doi:10.1016/j.apenergy.2016.11.030.

33. Sennott, T., Gotianun, C., Serres, R., Ziabasharhagh, M., Mack, J.H., and Dibble, R., "Artificial Neural Network for Predicting Cetane Number of Biofuel Candidates Based on Molecular 
Structure," Volume 2: Fuels; Numerical Simulation; Engine Design, Lubrication, and Applications, ASME, ISBN 978-0-7918-5610-9: V002T02A009, 2013, doi:10.1115/ICEF2013-19185.

34. Saldana, D.A., Starck, L., Mougin, P., Rousseau, B., Pidol, L., Jeuland, N., and Creton, B., "Flash Point and Cetane Number Predictions for Fuel Compounds Using Quantitative Structure Property Relationship (QSPR) Methods," Energy \& Fuels 25(9):3900-3908, 2011, doi:10.1021/ef200795j.

35. Creton, B., Dartiguelongue, C., Bruin, T. de, and Toulhoat, H., "Prediction of the Cetane Number of Diesel Compounds Using the Quantitative Structure Property Relationship," Energy \& Fuels 24(10):5396-5403, 2010, doi:10.1021/ef1008456.

36. Stratiev, D., Marinov, I., Dinkov, R., Shishkova, I., Velkov, I., Sharafutdinov, I., Nenov, S., Tsvetkov, T., Sotirov, S., Mitkova, M., and Rudnev, N., "Opportunity to Improve Diesel-Fuel Cetane-Number Prediction from Easily Available Physical Properties and Application of the Least-Squares Method and Artificial Neural Networks," Energy \& Fuels 29(3):1520-1533, 2015, doi:10.1021/ef502638c.

37. Abdul Jameel, A.G., Naser, N., Emwas, A.-H., Dooley, S., and Sarathy, S.M., "Predicting Fuel Ignition Quality Using 1 H NMR Spectroscopy and Multiple Linear Regression," Energy \& Fuels 30(11):9819-9835, 2016, doi:10.1021/acs.energyfuels.6b01690.

38. Sánchez-Borroto, Y., Piloto-Rodriguez, R., Errasti, M., Sierens, R., and Verhelst, S., "Prediction of cetane number and ignition delay of biodiesel using Artificial Neural Networks," Energy Procedia 57:877-885, 2014, doi:10.1016/j.egypro.2014.10.297.

39. Dahmen, M. and Marquardt, W., "A Novel Group Contribution Method for the Prediction of the Derived Cetane Number of Oxygenated Hydrocarbons," Energy \& Fuels 29(9):5781-5801, 2015, doi:10.1021/acs.energyfuels.5b01032.

40. Angikath, F., Naser, N., and Sarathy, S.M., "Investigating the effects of C3 and C4 alcohol blending on ignition quality of gasoline fuels," Energy \& Fuels acs.energyfuels.0c00192, 2020, doi:10.1021/acs.energyfuels.0c00192.

41. Heck, S.M., Pritchard, H.O., and Griffiths, J.F., "Cetane number vs. structure in paraffin hydrocarbons," J. Chem. Soc. Faraday Trans. 94(12):1725-1727, 1998, doi:10.1039/a800861b.

42. Lapidus, A.L., Smolenskii, E.A., Bavykin, V.M., Myshenkova, T.N., and Kondrat'ev, L.T., "Models for the calculation and prediction of the octane and cetane numbers of individual hydrocarbons," Pet. Chem. 48(4):277-286, 2008, doi:10.1134/\$0965544108040051.

43. DeFries, T.H., Kastrup, R. V., and Indritz, D., "Prediction of cetane number by group additivity and carbon-13 Nuclear Magnetic Resonance," Ind. Eng. Chem. Res. 26(2):188-193, 1987, doi:10.1021/ie00062a002.

44. Smolenskii, E.A., Bavykin, V.M., Ryzhov, A.N., Slovokhotova, O.L., Chuvaeva, I. V., and Lapidus, A.L., "Cetane numbers of hydrocarbons: calculations using optimal topological indices," Russ. Chem. Bull. 57(3):461-467, 2008, doi:10.1007/s11172-008-0073-0.

45. Nadai, D. V., Simões, J.B., Gatts, C.E.N., and Miranda, P.C.M.L., "Inference of the biodiesel cetane number by multivariate techniques," Fuel 105(x):325-330, 2013, doi:10.1016/j.fuel.2012.06.018.

46. Kubic, W.L., Jenkins, R.W., Moore, C.M., Semelsberger, T.A., and Sutton, A.D., "Artificial Neural Network Based Group Contribution Method for Estimating Cetane and Octane Numbers of Hydrocarbons and Oxygenated Organic Compounds," Ind. Eng. Chem. Res. 56(42):12236-12245, 
2017, doi:10.1021/acs.iecr.7b02753.

47. Schweidtmann, A.M., Rittig, J.G., König, A., Grohe, M., Mitsos, A., and Dahmen, M., "Graph Neural Networks for Prediction of Fuel Ignition Quality," Energy \& Fuels 34(9):11395-11407, 2020, doi:10.1021/acs.energyfuels.0c01533.

48. Souza, C.R., Silva, A.H., Nagata, N., Ribas, L.T., and Simonelli, F., "Cetane Number Assessment in Diesel Fuel by $1 \mathrm{H}$ or Hydrogen Nuclear Magnetic Resonance-Based Multivariate Calibration," 2014.

49. Kapur, G.S., Ecker, A., and Meusinger, R., "Establishing Quantitative Structure-Property Relationships (QSPR) of Diesel Samples by Proton-NMR \&amp; Multiple Linear Regression (MLR) Analysis," Energy \& Fuels 15(4):943-948, 2001, doi:10.1021/ef010021u.

50. Alves, J.C.L., Henriques, C.B., and Poppi, R.J., "Determination of diesel quality parameters using support vector regression and near infrared spectroscopy for an in-line blending optimizer system," Fuel 97:710-717, 2012, doi:10.1016/j.fuel.2012.03.016.

51. Pande, S.G. and Hardy, D.R., "Cetane number predictions of a trial index based on compositional analysis," Energy \& Fuels 3(3):308-312, 1989, doi:10.1021/ef00015a008.

52. Alizadeh, G., Frounchi, J., Baradaran Nia, M., Zarifi, M.H., and Asgarifar, S., "An FPGA implementation of an Artificial Neural Network for prediction of cetane number," 2008 International Conference on Computer and Communication Engineering, IEEE, ISBN 978-1-42441691-2: 605-608, 2008, doi:10.1109/ICCCE.2008.4580675.

53. Gulder, O.L. and Glavincevski, B., "Prediction of cetane number of diesel fuels from carbon type structural composition determined by proton NMR spectroscopy," Ind. Eng. Chem. Prod. Res. Dev. 25(2):153-156, 1986, doi:10.1021/i300022a005.

54. Ghosh, P. and Jaffe, S.B., "Detailed Composition-Based Model for Predicting the Cetane Number of Diesel Fuels," Ind. Eng. Chem. Res. 45(1):346-351, 2006, doi:10.1021/ie0508132.

55. Gülder, Ö.L., Burton, G.F., and Whyte, R.B., "NRCC Cetane Index - 1: An Improved Cetane Number Predictor," SAE Technical Paper, 12, 1986, doi:10.4271/861519.

56. Yang, H., Ring, Z., Briker, Y., McLean, N., Friesen, W., and Fairbridge, C., "Neural network prediction of cetane number and density of diesel fuel from its chemical composition determined by LC and GC-MS," Fuel 81(1):65-74, 2002, doi:10.1016/S0016-2361(01)00121-1.

57. Ladommatos, N. and Goacher, J., "Equations for predicting the cetane number of diesel fuels from their physical properties," Fuel 74(7):1083-1093, 1995, doi:10.1016/0016-2361(95)00040C.

58. Basu, B., Kapur, G.S., Sarpal, A.S., and Meusinger, R., "A Neural Network Approach to the Prediction of Cetane Number of Diesel Fuels Using Nuclear Magnetic Resonance (NMR) Spectroscopy," Energy \& Fuels 17(6):1570-1575, 2003, doi:10.1021/ef030083f.

59. O'Connor, C.T., Forrester, R.D., and Scurrell, M.S., "Cetane number determination of synthetic diesel fuels," Fuel 71(11):1323-1327, 1992, doi:10.1016/0016-2361(92)90061-R.

60. Piloto-Rodríguez, R., Sánchez-Borroto, Y., Lapuerta, M., Goyos-Pérez, L., and Verhelst, S., "Prediction of the cetane number of biodiesel using artificial neural networks and multiple linear 
regression," Energy Convers. Manag. 65:255-261, 2013, doi:10.1016/j.enconman.2012.07.023.

61. Yuan, H., Yang, B., and Yang, J., "Predicting Properties of Biodiesel Fuels using Mixture Topological Index," 2009, doi:10.1007/s11746-009-1354-y.

62. Hosseinpour, S., Aghbashlo, M., Tabatabaei, M., and Khalife, E., "Exact estimation of biodiesel cetane number (CN) from its fatty acid methyl esters (FAMEs) profile using partial least square (PLS) adapted by artificial neural network (ANN)," Energy Convers. Manag. 124:389-398, 2016, doi:https://doi.org/10.1016/j.enconman.2016.07.027.

63. Bolay, M., "Effect of viscosity on the cetane number of a diesel fuel," Masters Theses, 1953.

64. Abdul Jameel, A.G., "A functional group approach for predicting fuel properties," King Abdullah University of Science and Technology, 2019, doi:10.25781/KAUST-ZBB21.

65. Elbaz, A.M., Gani, A., Hourani, N., Emwas, A.-H., Sarathy, S.M., and Roberts, W.L., "TG/DTG, FTICR Mass Spectrometry, and NMR Spectroscopy Study of Heavy Fuel Oil," Energy \& Fuels 29(12):7825-7835, 2015, doi:10.1021/acs.energyfuels.5b01739.

66. Abdul Jameel, A.G., Oudenhoven, V. Van, Emwas, A.-H., and Sarathy, S.M., "Predicting Octane Number Using Nuclear Magnetic Resonance Spectroscopy and Artificial Neural Networks," Energy \& Fuels 32(5):6309-6329, 2018, doi:10.1021/acs.energyfuels.8b00556.

67. Lapuerta, M., Hernández, J.J., and Sarathy, S.M., "Effects of methyl substitution on the autoignition of C16 alkanes," Combust. Flame 164:259-269, 2016, doi:10.1016/j.combustflame.2015.11.024.

68. Naser, N., Abdul Jameel, A.G., Emwas, A.-H., Singh, E., Chung, S.H., and Sarathy, S.M., "The influence of chemical composition on ignition delay times of gasoline fractions," Combust. Flame 209:418-429, 2019, doi:10.1016/J.COMBUSTFLAME.2019.07.030.

69. Abdul Jameel, A.G., Naser, N., Issayev, G., Touitou, J., Ghosh, M.K., Emwas, A., Farooq, A., Dooley, S., and Sarathy, S.M., "A minimalist functional group (MFG) approach for surrogate fuel formulation," Combust. Flame 192:250-271, 2018, doi:10.1016/j.combustflame.2018.01.036.

70. Gani, A., Jameel, A., Naser, N., Emwas, A., and Sarathy, S.M., "Surrogate formulation for diesel and jet fuels using the minimalist functional group ( MFG ) approach," Proc. Combust. Inst. 37(4):4663-4671, 2019, doi:10.1016/j.proci.2018.09.035.

71. Mueller, C.J., Cannella, W.J., Bruno, T.J., Bunting, B., Dettman, H.D., Franz, J.A., Huber, M.L., Natarajan, M., Pitz, W.J., Ratcliff, M.A., and Wright, K., "Methodology for Formulating Diesel Surrogate Fuels with Accurate Compositional, Ignition-Quality, and Volatility Characteristics," Energy \& Fuels 26(6):3284-3303, 2012, doi:10.1021/ef300303e.

72. Mueller, C.J., Cannella, W.J., Bays, J.T., Bruno, T.J., DeFabio, K., Dettman, H.D., Gieleciak, R.M., Huber, M.L., Kweon, C.-B., McConnell, S.S., Pitz, W.J., and Ratcliff, M.A., "Diesel Surrogate Fuels for Engine Testing and Chemical-Kinetic Modeling: Compositions and Properties," Energy \& Fuels 30(2):acs.energyfuels.5b02879, 2016, doi:10.1021/acs.energyfuels.5b02879.

73. Dragoş Ilieş, B., Khandavilli, M., Li, Y., Kukkadapu, G., W. Wagnon, S., Gani Abdul Jameel, A., and Mani Sarathy, S., "Probing the Chemical Kinetics of Minimalist Functional Group Gasoline Surrogates," Energy \& Fuels 35(4):3315-3332, 2021, doi:10.1021/acs.energyfuels.0c02815. 
74. Yu, J., Ju, Y., and Gou, X., "Surrogate fuel formulation for oxygenated and hydrocarbon fuels by using the molecular structures and functional groups," Fuel 166:211-218, 2016, doi:10.1016/j.fuel.2015.10.085.

75. Mehl, M., Pitz, W.J., Sarathy, S.M., and Westbrook, C.K., "Modeling the combustion of high molecular weight fuels by a functional group approach," Int. J. Chem. Kinet. 44(4):257-276, 2012, doi:10.1002/kin.20715.

76. Won, S.H., Dooley, S., Veloo, P.S., Wang, H., Oehlschlaeger, M.A., Dryer, F.L., and Ju, Y., "The combustion properties of 2,6,10-trimethyl dodecane and a chemical functional group analysis," Combust. Flame 161(3):826-834, 2014, doi:10.1016/j.combustflame.2013.08.010.

77. Sumathi, R., Carstensen, H.-H., and Green, W.H., "Reaction Rate Prediction via Group Additivity Part 1: H Abstraction from Alkanes by H and CH 3," J. Phys. Chem. A 105(28):6910-6925, 2001, doi:10.1021/jp010697q.

78. Abdul Jameel, A.G., Han, Y., Brignoli, O., Telalović, S., Elbaz, A.M., Im, H.G., Roberts, W.L., and Sarathy, S.M., "Heavy fuel oil pyrolysis and combustion: Kinetics and evolved gases investigated by TGA-FTIR," J. Anal. Appl. Pyrolysis 127(August):183-195, 2017, doi:10.1016/j.jaap.2017.08.008.

79. Abdul Jameel, A.G. and Sarathy, S.M., "Prediction of RON and MON of gasoline-ethanol using $1 \mathrm{H}$ NMR spectroscopy," Proceedings of the European Combustion Meeting, 2017.

80. Abdul Jameel, A.G., Elbaz, A.M., Emwas, A.-H., Roberts, W.L., and Sarathy, S.M., "Calculation of Average Molecular Parameters, Functional Groups, and a Surrogate Molecule for Heavy Fuel Oils Using $1 \mathrm{H}$ and 13 C Nuclear Magnetic Resonance Spectroscopy," Energy \& Fuels 30(5):3894-3905, 2016, doi:10.1021/acs.energyfuels.6b00303.

81. Abdul Jameel, A.G. and Sarathy, S.M., "Lube Products: Molecular Characterization of Base Oils," Encyclopedia of Analytical Chemistry, John Wiley \& Sons, Ltd, Chichester, UK, ISBN 0471976709, 9780471976707, 0470027312, 9780470027318: 1-14, 2018, doi:10.1002/9780470027318.a1824.pub2.

82. Campuzano, F., Abdul Jameel, A.G., Zhang, W., Emwas, A.-H., Agudelo, A.F., Martínez, J.D., and Sarathy, S.M., "Fuel and Chemical Properties of Waste Tire Pyrolysis Oil Derived from a Continuous Twin-Auger Reactor," Energy \& Fuels 34(10):12688-12702, 2020, doi:10.1021/acs.energyfuels.0c02271.

83. Campuzano, F., Abdul Jameel, A.G., Zhang, W., Emwas, A., Agudelo, A.F., Martínez, J.D., and Sarathy, S.M., "On the distillation of waste tire pyrolysis oil: A structural characterization of the derived fractions," Fuel 290(August 2020):120041, 2021, doi:10.1016/j.fuel.2020.120041.

84. Abdul Jameel, A.G., Khateeb, A., Elbaz, A.M., Emwas, A.-H., Zhang, W., Roberts, W.L., and Sarathy, S.M., "Characterization of deasphalted heavy fuel oil using APPI (+) FT-ICR mass spectrometry and NMR spectroscopy," Fuel 253:950-963, 2019, doi:10.1016/j.fuel.2019.05.061.

85. Dahmen, M., Hechinger, M., Victoria Villeda, J., and Marquardt, W., "Towards Model-Based Identification of Biofuels for Compression Ignition Engines," SAE Int. J. Fuels Lubr. 5(3):2012-011593, 2012, doi:10.4271/2012-01-1593.

86. Maylin, M.V., Kirgina, M.V., Sviridova, E.V., Sakhnevitch, B.V., and Ivanchina, E.D., "Calculation of Gasoline Octane Numbers Taking into Account the Reaction Interaction of Blend Components," 
Procedia Chem. 10:477-484, 2014, doi:10.1016/j.proche.2014.10.080.

87. AlRamadan, A.S., Sarathy, S.M., Khurshid, M., and Badra, J., "A blending rule for octane numbers of PRFs and TPRFs with ethanol," Fuel 180(March 2007):175-186, 2016, doi:10.1016/j.fuel.2016.04.032.

88. Morgan, N., Smallbone, A., Bhave, A., Kraft, M., Cracknell, R., and Kalghatgi, G., "Mapping surrogate gasoline compositions into RON/MON space," Combust. Flame 157(6):1122-1131, 2010, doi:10.1016/j.combustflame.2010.02.003.

89. Foong, T.M., Morganti, K.J., Brear, M.J., Silva, G. da, Yang, Y., and Dryer, F.L., "The octane numbers of ethanol blended with gasoline and its surrogates," Fuel 115:727-739, 2014, doi:10.1016/j.fuel.2013.07.105.

90. Kathrotia, T. and Riedel, U., "Predicting the soot emission tendency of real fuels - A relative assessment based on an empirical formula," Fuel 261:116482, 2020, doi:10.1016/J.FUEL.2019.116482.

91. Andrae, J.C.G., "Development of a detailed kinetic model for gasoline surrogate fuels," Fuel 87(10-11):2013-2022, 2008, doi:10.1016/j.fuel.2007.09.010.

92. Badra, J., AlRamadan, A.S., and Sarathy, S.M., "Optimization of the octane response of gasoline/ethanol blends," Appl. Energy 203(March 2007):778-793, 2017, doi:10.1016/j.apenergy.2017.06.084.

93. Yang, S.Y., Naser, N., Chung, S.H., and Cha, J., "Effect of Temperature, Pressure and Equivalence Ratio on Ignition Delay in Ignition Quality Tester (IQT): Diesel, $\mathrm{n}$-Heptane, and iso -Octane Fuels under Low Temperature Conditions," SAE Int. J. Fuels Lubr. 8(3):2015-01-9074, 2015, doi:10.4271/2015-01-9074.

94. Waqas, M., Naser, N., Sarathy, M., Feijs, J., Morganti, K., Nyrenstedt, G., and Johansson, B., "Auto-Ignition of Iso-Stoichiometric Blends of Gasoline-Ethanol-Methanol (GEM) in SI, HCCI and CI Combustion Modes," SAE International, ISBN 0148-7191, 2017, doi:10.4271/2017-01-0726.

95. Waqas, M., Naser, N., Sarathy, M., Morganti, K., Al-Qurashi, K., and Johansson, B., "Blending Octane Number of Ethanol in $\mathrm{HCCl}, \mathrm{SI}$ and $\mathrm{Cl}$ Combustion Modes," SAE Int. J. Fuels Lubr. 9(3):2016-01-2298, 2016, doi:10.4271/2016-01-2298.

96. Nicolle, A., Naser, N., Javed, T., Rankovic, N., and Sarathy, S.M., "Autoignition Characteristics of Ethers Blended with Low Cetane Distillates," Energy \& Fuels 33(7):6775-6787, 2019, doi:10.1021/acs.energyfuels.9b00571.

97. Blondel-Telouk, A., Loiseleur, H., Barreau, A., Behar, E., and Jose, J., "Determination of the average molecular weight of petroleum cuts by vapor pressure depression," Fluid Phase Equilib. 110(1):315-339, 1995, doi:https://doi.org/10.1016/0378-3812(95)02760-C.

98. Sarowha, S.L.S., "Determination of Molecular Weights of Petroleum Products Using Gel Permeation Chromatography," Pet. Sci. Technol. 23(5-6):573-578, 2005, doi:10.1081/LFT200032846.

99. Rao, V.K. and Bardon, M.F., "Estimating the molecular weight of petroleum fractions," Ind. Eng. Chem. Process Des. Dev. 24(2):498-500, 1985, doi:10.1021/i200029a046. 
100. Kang, D., Kirby, S., Agudelo, J., Lapuerta, M., Al-Qurashi, K., and Boehman, A.L., "Combined Impact of Branching and Unsaturation on the Autoignition of Binary Blends in a Motored Engine," Energy \& Fuels 28(11):7203-7215, 2014, doi:10.1021/ef501629p.

101. Sarathy, S.M., Javed, T., Karsenty, F., Heufer, A., Wang, W., Park, S., Elwardany, A., Farooq, A., Westbrook, C.K., Pitz, W.J., Oehlschlaeger, M.A., Dayma, G., Curran, H.J., and Dagaut, P., "A comprehensive combustion chemistry study of 2,5-dimethylhexane," Combust. Flame 161(6):1444-1459, 2014, doi:10.1016/j.combustflame.2013.12.010.

102. Santana, R.C., Do, P.T., Santikunaporn, M., Alvarez, W.E., Taylor, J.D., Sughrue, E.L., and Resasco, D.E., "Evaluation of different reaction strategies for the improvement of cetane number in diesel fuels," Fuel 85(5-6):643-656, 2006, doi:10.1016/j.fuel.2005.08.028.

103. Sarathy, S.M., Yeung, C., Westbrook, C.K., Pitz, W.J., Mehl, M., and Thomson, M.J., "An experimental and kinetic modeling study of n-octane and 2-methylheptane in an opposed-flow diffusion flame," Combust. Flame 158(7):1277-1287, 2011, doi:10.1016/j.combustflame.2010.11.008.

104. N. Srivastava, G. Hinton, A. Krizhevsky, I. Sutskever, R. Salakhutdinov, Y.B., "Dropout: A Simple Way to Prevent Neural Networks from Overfittin," J. Mach. Learn. Res. 15:1929-1958, 2014.

105. Chollet, F., "Xception: Deep Learning with Depthwise Separable Convolutions," 2017 IEEE Conference on Computer Vision and Pattern Recognition (CVPR), IEEE, ISBN 978-1-5386-0457-1: 1800-1807, 2017, doi:10.1109/CVPR.2017.195.

106. Abadi, M., Agarwal, A., Barham, P., Brevdo, E., Chen, Z., Citro, C., Corrado, G.S., Davis, A., Dean, J., Devin, M., Ghemawat, S., Goodfellow, I., Harp, A., Irving, G., Isard, M., Jia, Y., Jozefowicz, R., Kaiser, L., Kudlur, M., Levenberg, J., Mane, D., Monga, R., Moore, S., Murray, D., Olah, C., Schuster, M., Shlens, J., Steiner, B., Sutskever, I., et al., "TensorFlow: Large-Scale Machine Learning on Heterogeneous Distributed Systems," 2016.

107. Kingma, D.P. and Ba, J., “Adam: A Method for Stochastic Optimization," 2014, doi:http://doi.acm.org.ezproxy.lib.ucf.edu/10.1145/1830483.1830503.

108. He, K., Zhang, X., Ren, S., and Sun, J., "Delving Deep into Rectifiers: Surpassing Human-Level Performance on ImageNet Classification," 2015 IEEE International Conference on Computer Vision (ICCV), ISBN 2380-7504 VO -: 1026-1034, 2015, doi:10.1109/ICCV.2015.123.

109. Nair, V. and Hinton, G.E., "Rectified Linear Units Improve Restricted Boltzmann Machines," ICML, 2010.

110. Brocard, J.C., Baronnet, F., and O'Neal, H.E., "Chemical kinetics of the oxidation of methyl tertbutyl ether (MTBE)," Combust. Flame 52(C):25-35, 1983, doi:10.1016/0010-2180(83)90118-9.

111. Kadi, B. El and Baronnet, F., "Study of the oxidation of unsymmetrical ethers (ETBE, TAME) and tentative interpretation of their high octane numbers," J. Chim. Phys. 92:706-725, 1995, doi:10.1051/jcp/1995920706.

112. Fischer, S.L., Dryer, F.L., and Curran, H.J., "The reaction kinetics of dimethyl ether. I: Hightemperature pyrolysis and oxidation in flow reactors," Int. J. Chem. Kinet. 32(12):713-740, 2000, doi:10.1002/1097-4601(2000)32:12<713::AID-KIN1>3.0.CO;2-9.

113. Choo, K.Y., Golden, D.M., and Benson, S.W., "Very low-pressure pyrolysis (VLPP) oft-butylmethyl 
ether," Int. J. Chem. Kinet. 6(5):631-641, 1974, doi:10.1002/kin.550060503.

114. Liotta, F.J. and Montalvo, D.M., "The Effect of Oxygenated Fuels on Emissions from a Modern Heavy-Duty Diesel Engine," SAE International, 1993, doi:10.4271/932734.

115. Sivasankaralingam, V., Raman, V., Mubarak Ali, M.J., Alfazazi, A., Lu, T., Im, H., Sarathy, S.M., and Dibble, R., "Experimental and Numerical Investigation of Ethanol/Diethyl Ether Mixtures in a $\mathrm{Cl}$ Engine," SAE International, 2016, doi:10.4271/2016-01-2180.

116. Cai, L., Sudholt, A., Lee, D.J., Egolfopoulos, F.N., Pitsch, H., Westbrook, C.K., and Sarathy, S.M., "Chemical kinetic study of a novel lignocellulosic biofuel: Di-n-butyl ether oxidation in a laminar flow reactor and flames," Combust. Flame 161(3):798-809, 2014, doi:10.1016/j.combustflame.2013.10.003. 


\section{Supplementary Material}

\section{Predicting ignition quality of oxygenated fuels using artificial neural networks}

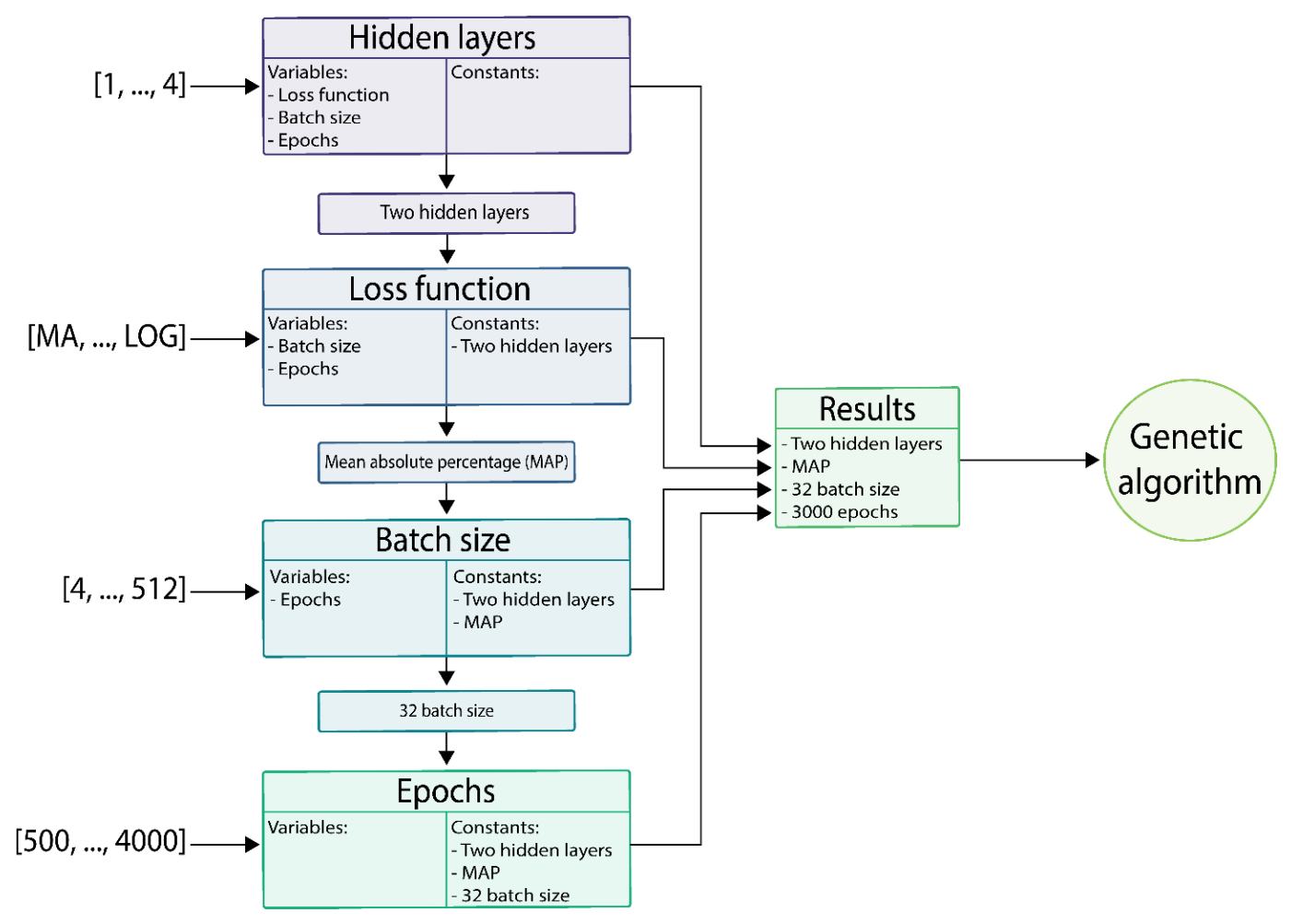

Fig. S1. High level overview of the hyperparameter tuning procedure

\section{Grid search and genetic algorithm}

The originally split train set was used as the dataset and the fitness is the average percentage error over the 5 folds. Once each individual has been evaluated, they are ranked based on their fitness. A new population is generated through two methods: crossover and mutation. The best performing parents are used in order to create new individuals. This is done by taking two parents, initializing the individual's parameters to that of the best parent and then modifying a single parameter based on the value of the other parent. Once 20 individuals have been created through crossover certain individuals are mutated - a single parameter is modified randomly, limited by the same constraints. This is repeated 
for 15 generations. The architecture of the fittest individual is used to create the final model. Fig. S2. illustrates the entire process. Each ANN is represented by an oval containing four horizontal bars, which depict the values of the tweaked parameters.

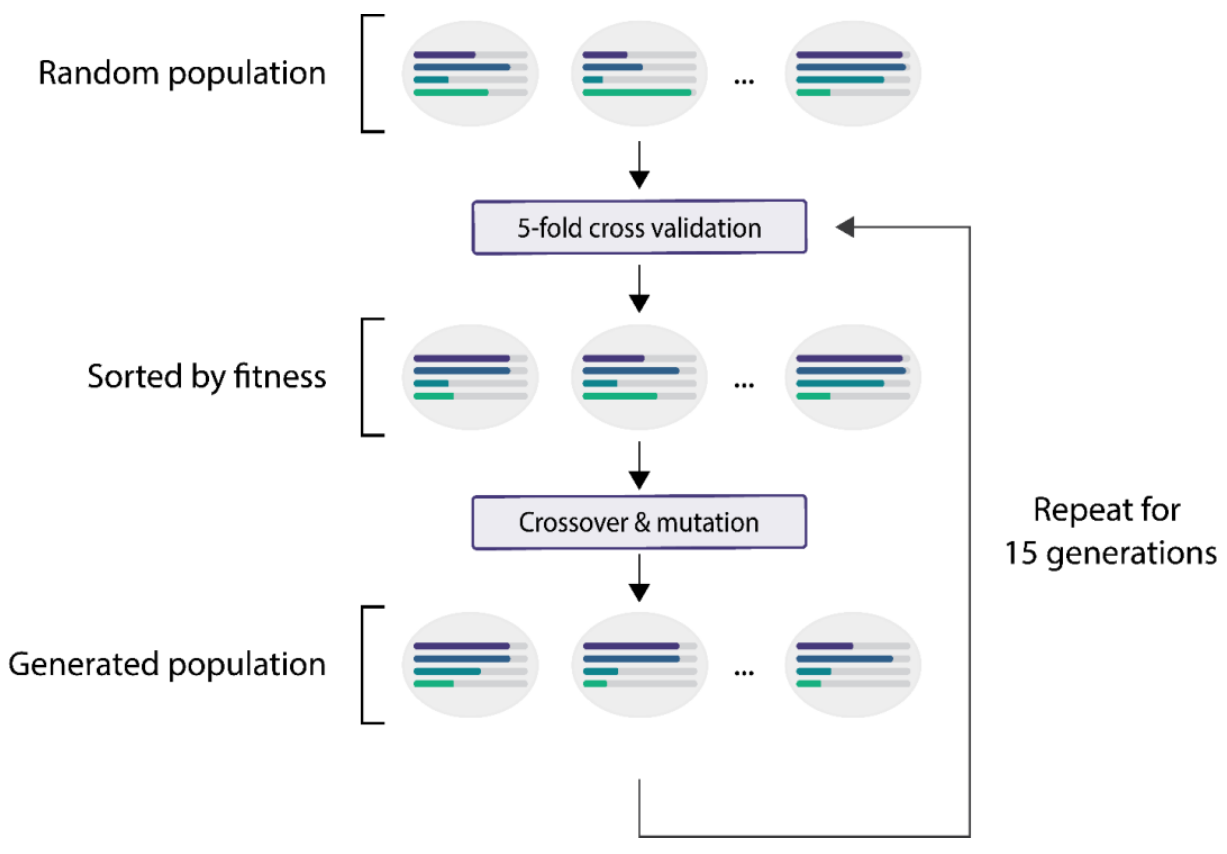

Fig. S2. Steps taken by the genetic algorithm to develop the final ANN mode 
